Supplement of Hydrol. Earth Syst. Sci., 20, 3719-3737, 2016

http://www.hydrol-earth-syst-sci.net/20/3719/2016/

doi:10.5194/hess-20-3719-2016-supplement

(c) Author(s) 2016. CC Attribution 3.0 License.

(c) (1)

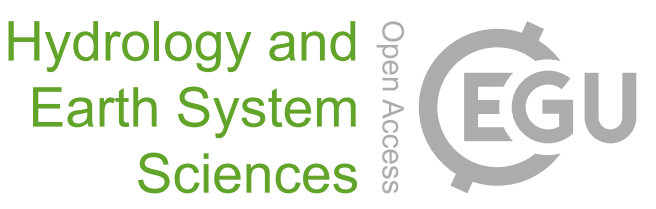

Supplement of

\title{
Use of column experiments to investigate the fate of organic micropollutants - a review
}

Stefan Banzhaf and Klaus H. Hebig

Correspondence to: Stefan Banzhaf (stefan.banzhaf@gu.se)

The copyright of individual parts of the supplement might differ from the CC-BY 3.0 licence. 


\begin{tabular}{|c|c|c|c|c|c|c|c|c|c|c|c|c|c|c|c|c|}
\hline \multirow[b]{2}{*}{ Citation } & \multirow{2}{*}{$\begin{array}{l}\text { Condi- } \\
\text { tions }\end{array}$} & \multirow[b]{2}{*}{ Compounds } & \multicolumn{3}{|c|}{ Column } & \multicolumn{3}{|c|}{ Sediment } & \multirow{2}{*}{$\begin{array}{l}\text { Velocities / } \\
\text { flowrates }\end{array}$} & \multirow{2}{*}{$\begin{array}{l}\text { Filter } \\
\text { layers }\end{array}$} & \multicolumn{3}{|c|}{ Infrastructure / used materials } & \multirow{2}{*}{$\begin{array}{l}\text { Input } \\
\text { concen- } \\
\text { trations }\end{array}$} & \multirow{2}{*}{$\begin{array}{l}\text { Model- } \\
\text { ling }\end{array}$} & \multirow{2}{*}{$\begin{array}{l}\text { Sampling } \\
\text { methods }\end{array}$} \\
\hline & & & material & $\begin{array}{l}\text { length } \\
{[\mathrm{cm}]}\end{array}$ & $\begin{array}{l}\text { diameter } \\
{[\mathrm{cm}]}\end{array}$ & type & $\begin{array}{l}\text { instal- } \\
\text { lation }\end{array}$ & porosity & & & Tubes & Pump & Fluids & & & \\
\hline $\begin{array}{l}\text { Alidina et al. } \\
\text { (2014) }\end{array}$ & $\begin{array}{l}\text { saturated } \\
\text { flow } \\
\text { through }\end{array}$ & $\begin{array}{l}\text { atenolol, } \\
\text { caffeine, } \\
\text { diclofenac, } \\
\text { gemfibrozil, } \\
\text { primidone, } \\
\text { trimethoprim }\end{array}$ & glass & $\begin{array}{l}4 \times 100 \\
\text { (in se- } \\
\text { ries) }\end{array}$ & 5 & natural soil & $\begin{array}{l}\text { sieved, } \\
\text { wet } \\
\text { (satu- } \\
\text { rated) }\end{array}$ & 0.32 & $\begin{array}{l}1.44 \mathrm{~m} / \mathrm{d} \\
\text { (as "loading } \\
\text { rate") }\end{array}$ & $\mathrm{n} / \mathrm{a}$ & $\mathrm{n} / \mathrm{a}$ & $\begin{array}{l}\text { peristal- } \\
\text { tic pump }\end{array}$ & $\begin{array}{l}\text { synthetic } \\
\text { wastewater with } \\
\text { different ratios of } \\
\text { different organic } \\
\text { carbon sources }\end{array}$ & $\begin{array}{l}300-500 \\
\mathrm{ng} / \mathrm{L}\end{array}$ & $\mathrm{n} / \mathrm{a}$ & $\begin{array}{l}\text { sampling } \\
\text { ports }\end{array}$ \\
\hline $\begin{array}{l}\text { Alotaibi et al. } \\
\text { (2015) }\end{array}$ & $\begin{array}{l}\text { saturated } \\
\text { flow } \\
\text { through }\end{array}$ & $\begin{array}{l}\text { 5-methylbenzotriazole, } \\
\text { benzotriazole }\end{array}$ & $\begin{array}{l}\text { stainless } \\
\text { steel }\end{array}$ & 200 & 14.5 & $\begin{array}{l}\text { natural } \\
\text { sediment }\end{array}$ & $\begin{array}{l}\text { wet } \\
\text { (satu- } \\
\text { rated) } \\
\text { under } \\
\text { flushing } \\
\text { nitro- } \\
\text { gen } \\
\text { gas }\end{array}$ & $\begin{array}{l}0.402 \\
\text { (bromide } \\
\text { tracer } \\
\text { test) }\end{array}$ & $\begin{array}{l}2.86 \mathrm{~cm} / \mathrm{d} \\
\text { (bromide } \\
\text { tracer test) }\end{array}$ & $\mathrm{n} / \mathrm{a}$ & $n / a$ & $\begin{array}{l}\text { gear } \\
\text { pump }\end{array}$ & $\begin{array}{l}\text { treated waste wa- } \\
\text { ter }\end{array}$ & $\begin{array}{l}200 \mathrm{ng} / \mathrm{L} \\
\text { per com- } \\
\text { pound }\end{array}$ & $\begin{array}{l}\text { Origin } \\
\text { v61 } \\
\text { (CDE) }\end{array}$ & $\begin{array}{l}\text { sampling } \\
\text { ports }\end{array}$ \\
\hline $\begin{array}{l}\text { Banzhaf et al. } \\
\text { (2012) }\end{array}$ & $\begin{array}{l}\text { saturated } \\
\text { flow } \\
\text { through }\end{array}$ & $\begin{array}{l}\text { carbamazepine, } \\
\text { diclofenac, } \\
\text { ibuprofen, } \\
\text { sulfamethoxazole }\end{array}$ & $\begin{array}{l}\text { stainless } \\
\text { steel }\end{array}$ & 35 & 13.6 & $\begin{array}{l}\text { natural } \\
\text { sediment }\end{array}$ & $\begin{array}{l}\text { dry, } \\
\text { com- } \\
\text { paction } \\
\text { by a } \\
\text { stamp }\end{array}$ & $\begin{array}{l}0.42 \text { (to- } \\
\text { tal) } \\
0.41 \text { (ef- } \\
\text { fective } \\
\text { from } \\
\text { tracer ex- } \\
\text { periment) }\end{array}$ & $\begin{array}{l}14 \mathrm{~mL} / \mathrm{h} / \\
6.3-6.5 \\
\mathrm{~cm} / \mathrm{d}\end{array}$ & $\begin{array}{l}\text { quartz } \\
\text { filter } \\
\text { sand / } \\
\text { gravel }\end{array}$ & $n / a$ & $\begin{array}{l}\text { peristal- } \\
\text { tic pump }\end{array}$ & $\begin{array}{l}\text { natural surface } \\
\text { water }\end{array}$ & $\begin{array}{l}165-295 \\
\mu \mathrm{g} / \mathrm{L}\end{array}$ & $\begin{array}{l}\text { CXTFIT } \\
\text { (Toride } \\
\text { et al., } \\
1995 \text { ) }\end{array}$ & $\begin{array}{l}\text { flowthrough } \\
\text { cells (phys- } \\
\text { ico-chemical } \\
\text { parameters), } \\
\text { fraction col- } \\
\text { lector }\end{array}$ \\
\hline $\begin{array}{l}\text { Baumgarten } \\
\text { et al. (2011) }\end{array}$ & $\begin{array}{l}\text { saturated } \\
\text { flow } \\
\text { through }\end{array}$ & sulfamethoxazole & $n / a$ & 200 & 15.5 & $\begin{array}{l}\text { artificial } \\
\text { sediment } \\
\text { (filter } \\
\text { sand/tech- } \\
\text { nical } \\
\text { quartz } \\
\text { sand), } 0.7 \\
-1.2 \mathrm{~mm} \\
\text { grain size }\end{array}$ & $\mathrm{n} / \mathrm{a}$ & $\begin{array}{l}0.4 \\
\text { (“void- } \\
\text { age") }\end{array}$ & $\begin{array}{l}0.13 \mathrm{~m} / \mathrm{d} \\
\left(\mathrm{v}_{\mathrm{f}}\right)\end{array}$ & $\mathrm{n} / \mathrm{a}$ & $\mathrm{n} / \mathrm{a}$ & $\mathrm{n} / \mathrm{a}$ & $\begin{array}{l}\text { natural surface } \\
\text { water (aerobic \& } \\
\text { anaerobic) }\end{array}$ & $\begin{array}{l}0.25- \\
4.15 \mu \mathrm{g} / \mathrm{L}\end{array}$ & $\mathrm{n} / \mathrm{a}$ & $\begin{array}{l}\text { sampling } \\
\text { ports (online: } \\
\text { pH, O2; of- } \\
\text { fline: } 0.2 \mathrm{~L} \\
\text { samples), } \\
0.2 \mathrm{~L} \text { sam- } \\
\text { ples at the in- } \\
\text { fluent \& efflu- } \\
\text { ent }\end{array}$ \\
\hline $\begin{array}{l}\text { Bertelkamp et } \\
\text { al. (2012) }\end{array}$ & $\begin{array}{l}\text { saturated } \\
\text { flow } \\
\text { through }\end{array}$ & $\begin{array}{l}\text { acetaminophen, } \\
\text { atrazine, } \\
\text { caffeine, } \\
\text { carbamazepine, } \\
\text { gemfibrozil, } \\
\text { ibuprofen, } \\
\text { hydrochlorothiazide, } \\
\text { Ketoprofen, } \\
\text { lincomycin, } \\
\text { phenytoin, } \\
\text { propranolol, } \\
\text { metoprolol, } \\
\text { trimethoprim, } \\
\text { sulfamethoxazole }\end{array}$ & $\begin{array}{l}\text { PVC } \\
\text { (transpar- } \\
\text { ent) }\end{array}$ & 100 & 3.6 & $\begin{array}{l}\text { artificial } \\
\text { sediment } \\
\text { (filter } \\
\text { sand/tech- } \\
\text { nical } \\
\text { quartz } \\
\text { sand), } 1.4 \\
-2 \mathrm{~mm}\end{array}$ & $\mathrm{n} / \mathrm{a}$ & $0.31-0.4$ & $\begin{array}{l}2.4-3.2 \\
\text { m/d (pore } \\
\text { velocity, } \\
\text { NaCl } \\
\text { Tracer) }\end{array}$ & $\mathrm{n} / \mathrm{a}$ & $\begin{array}{l}\text { dark pol- } \\
\text { yethylene } \\
\text {, Mar- } \\
\text { prene® } \\
\text { pump } \\
\text { tubing }\end{array}$ & $\begin{array}{l}\text { peristal- } \\
\text { tic pump }\end{array}$ & $\begin{array}{l}\text { natural surface } \\
\text { water with sodium } \\
\text { acetate or sodium } \\
\text { azide, tap water } \\
\text { spiked with OMPs }\end{array}$ & $\begin{array}{l}200 \mathrm{ng} / \mathrm{L} \\
\text { per sub- } \\
\text { stance }\end{array}$ & $\mathrm{n} / \mathrm{a}$ & bottles \\
\hline
\end{tabular}




\begin{tabular}{|c|c|c|c|c|c|c|c|c|c|c|c|c|c|c|c|c|}
\hline \multirow{2}{*}{ Citation } & \multirow{2}{*}{$\begin{array}{l}\text { Condi- } \\
\text { tions }\end{array}$} & \multirow{2}{*}{ Compounds } & \multicolumn{3}{|c|}{ Column } & \multicolumn{3}{|c|}{ Sediment } & \multirow{2}{*}{\begin{tabular}{|l} 
Velocities / \\
flowrates
\end{tabular}} & \multirow{2}{*}{$\begin{array}{l}\text { Filter } \\
\text { layers }\end{array}$} & \multicolumn{3}{|c|}{ Infrastructure / used materials } & \multirow{2}{*}{\begin{tabular}{|c|} 
Input \\
concen- \\
trations
\end{tabular}} & \multirow{2}{*}{$\begin{array}{l}\text { Model- } \\
\text { ling }\end{array}$} & \multirow{2}{*}{$\begin{array}{l}\text { Sampling } \\
\text { methods }\end{array}$} \\
\hline & & & material & $\begin{array}{l}\text { length } \\
{[\mathrm{cm}]}\end{array}$ & $\begin{array}{c}\text { diameter } \\
{[\mathrm{cm}]}\end{array}$ & type & $\begin{array}{l}\text { instal- } \\
\text { lation }\end{array}$ & porosity & & & Tubes & Pump & Fluids & & & \\
\hline $\begin{array}{l}\text { Bertelkamp et } \\
\text { al. (2014) }\end{array}$ & $\begin{array}{l}\text { saturated } \\
\text { flow } \\
\text { through }\end{array}$ & $\begin{array}{l}\text { acetaminophen, } \\
\text { atrazine, } \\
\text { caffeine, } \\
\text { carbamazepine, } \\
\text { gemfibrozil, } \\
\text { ibuprofen, } \\
\text { hydrochlorothiazide, } \\
\text { ketoprofen, } \\
\text { lincomycin, } \\
\text { phenytoin, } \\
\text { propranolol, } \\
\text { metoprolol, } \\
\text { trimethoprim, } \\
\text { sulfamethoxazole }\end{array}$ & \begin{tabular}{|l|} 
PVC \\
(transpar- \\
ent)
\end{tabular} & 100 & 3.6 & $\begin{array}{l}\text { artificial } \\
\text { sediment } \\
\text { (filter } \\
\text { sand/tech- } \\
\text { nical } \\
\text { quartz } \\
\text { sand), } 1.4 \\
-2 \mathrm{~mm}\end{array}$ & $\begin{array}{l}\text { com- } \\
\text { paction } \\
\text { by tap- } \\
\text { ping in } \\
4-5 \\
\mathrm{~cm} \text { in- } \\
\text { cre- } \\
\text { ments }\end{array}$ & $\begin{array}{l}0.31 \\
0.42\end{array}$ & $\begin{array}{l}2.4-3.2 \\
\mathrm{~m} / \mathrm{d}\end{array}$ & $\begin{array}{l}\text { perfo- } \\
\text { rated } \\
\text { PVC } \\
\text { plates }\end{array}$ & $\begin{array}{l}\text { dark pol- } \\
\text { yethylene } \\
\text {, Mar- } \\
\text { prene® } \\
\text { pump } \\
\text { tubing }\end{array}$ & $\begin{array}{l}\text { peristal- } \\
\text { tic pump }\end{array}$ & $\begin{array}{l}\text { natural surface } \\
\text { water with sodium } \\
\text { acetate or sodium } \\
\text { azide, demineral- } \\
\text { ized water }\end{array}$ & \begin{tabular}{|l}
$200 \mathrm{ng} / \mathrm{L}$ \\
per sub- \\
stance
\end{tabular} & $\begin{array}{l}\text { CXTFIT } \\
\text { (Toride } \\
\text { et al., } \\
1995 \text { ) }\end{array}$ & bottles \\
\hline $\begin{array}{l}\text { Burke et al. } \\
(2013)\end{array}$ & $\begin{array}{l}\text { saturated } \\
\text { flow } \\
\text { through }\end{array}$ & $\begin{array}{l}\text { 1-acetyl-1-methyl-2-di- } \\
\text { methyl-oxamoyl-2-phe- } \\
\text { nylhydrazide, } \\
\text { 1-acetyl-1-methyl-2-phe- } \\
\text { nylhydrazide, } \\
\text { acetylaminoantipyrine, } \\
\text { atenolol, } \\
\text { diazepam, } \\
\text { formylaminoantipyrine, } \\
\text { meprobamate, } \\
\text { metoprolol, } \\
\text { N-methylphenacetine, } \\
\text { oxazepam, } \\
\text { p-TSA, } \\
\text { phenacetine, } \\
\text { phenazone, } \\
\text { phenylethylmalonamide, } \\
\text { primidone, } \\
\text { propranolol, } \\
\text { propyphenazone, } \\
\text { pyrithylidone, } \\
\text { sotalol, } \\
\text { tolytriazole, }\end{array}$ & $\begin{array}{l}\text { stainless } \\
\text { steel }\end{array}$ & 30 & 10 & $\begin{array}{l}\text { natural } \\
\text { sediment }\end{array}$ & $\begin{array}{l}\text { wet } \\
\text { (satu- } \\
\text { rated), } \\
\text { com- } \\
\text { paction } \\
\text { by vi- } \\
\text { bration }\end{array}$ & 0.45 & $0.147 \mathrm{~m} / \mathrm{d}$ & $\begin{array}{l}\text { stain- } \\
\text { less } \\
\text { steel } \\
\text { mesh }\end{array}$ & PTFE & $\mathrm{n} / \mathrm{a}$ & tap water & $\begin{array}{l}0.8 \mu \mathrm{g} / \mathrm{L} \\
\text { each } \\
\text { com- } \\
\text { pound }\end{array}$ & $\begin{array}{l}\text { Phreeq } \\
\text { c-2 } \\
\text { (Parkhu } \\
\text { rst and } \\
\text { Appelo, } \\
\text { 1999) }\end{array}$ & $\begin{array}{l}\text { fraction col- } \\
\text { lector }\end{array}$ \\
\hline $\begin{array}{l}\text { Burke et al. } \\
(2014)\end{array}$ & $\begin{array}{l}\text { saturated } \\
\text { flow } \\
\text { through }\end{array}$ & $\begin{array}{l}\text { acesulfame, } \\
\text { acetylaminoantipyrine, } \\
\text { benzotriazole, } \\
\text { carbamazepine, } \\
\text { diclofenac, } \\
\text { formylaminoantipyrine, } \\
\text { iopromide, } \\
\text { metoprolol, } \\
\text { phenazone, } \\
\text { tolyltriazole, }\end{array}$ & aluminum & 120 & 8 & $\begin{array}{l}\text { natural } \\
\text { sediment }\end{array}$ & $\begin{array}{l}\text { undis- } \\
\text { turbed } \\
\text { core }\end{array}$ & 0.3 & $\begin{array}{l}1.38 \mathrm{~m} / \mathrm{d} \\
\text { (pore water } \\
\text { velocity) }\end{array}$ & $\mathrm{n} / \mathrm{a}$ & $n / a$ & $\begin{array}{l}\text { peristal- } \\
\text { tic pump }\end{array}$ & $\begin{array}{l}\text { natural surface } \\
\text { water }\end{array}$ & $\begin{array}{l}0.07- \\
0.72 \\
\mu \mathrm{g} / \mathrm{L}\end{array}$ & $\begin{array}{l}\text { PEST, } \\
\text { removal } \\
\text { rates } \\
\text { (Dohert } \\
\text { y, 2005) }\end{array}$ & $\begin{array}{l}\text { sampling } \\
\text { ports, by } \\
\text { hand }\end{array}$ \\
\hline
\end{tabular}




\begin{tabular}{|c|c|c|c|c|c|c|c|c|c|c|c|c|c|c|c|c|}
\hline \multirow{2}{*}{ Citation } & \multirow{2}{*}{$\begin{array}{l}\text { Condi- } \\
\text { tions }\end{array}$} & \multirow{2}{*}{ Compounds } & \multicolumn{3}{|c|}{ Column } & \multicolumn{3}{|c|}{ Sediment } & \multirow{2}{*}{$\begin{array}{l}\text { Velocities / } \\
\text { flowrates }\end{array}$} & \multirow{2}{*}{$\begin{array}{l}\text { Filter } \\
\text { layers }\end{array}$} & \multicolumn{3}{|c|}{ Infrastructure / used materials } & \multirow{2}{*}{$\begin{array}{l}\text { Input } \\
\text { concen- } \\
\text { trations }\end{array}$} & \multirow{2}{*}{$\begin{array}{l}\text { Model- } \\
\text { ling }\end{array}$} & \multirow{2}{*}{$\begin{array}{l}\text { Sampling } \\
\text { methods }\end{array}$} \\
\hline & & & material & $\begin{array}{c}\text { length } \\
{[\mathrm{cm}]}\end{array}$ & $\begin{array}{l}\text { diameter } \\
{[\mathrm{cm}]}\end{array}$ & type & $\begin{array}{l}\text { instal- } \\
\text { lation }\end{array}$ & porosity & & & Tubes & Pump & Fluids & & & \\
\hline $\begin{array}{l}\text { Burke et al. } \\
(2016)\end{array}$ & $\begin{array}{l}\text { saturated } \\
\text { flow } \\
\text { through }\end{array}$ & $\begin{array}{l}\text { atenolol, } \\
\text { propranolol, } \\
\text { metoprolol, } \\
\text { sotalol, } \\
\text { iopromide, } \\
\text { phenazone, } \\
\text { propyphenazone, } \\
\text { FAA, } \\
\text { AAA, } \\
\text { AMPH, } \\
\text { AMDOPH, } \\
\text { diazepam, } \\
\text { oxazepam, } \\
\text { primidone, } \\
\text { PEMA, } \\
\text { carbamazepine, } \\
\text { trimethoprim, } \\
\text { benzotriazole, } \\
\text { tolyltriazole, } \\
\text { acesulfame }\end{array}$ & \begin{tabular}{|l|} 
stainless \\
steel
\end{tabular} & 30 & 10 & $\begin{array}{l}\text { natural } \\
\text { sediment }\end{array}$ & $\begin{array}{l}\text { wet } \\
\text { (satu- } \\
\text { rated), } \\
\text { com-- } \\
\text { paction } \\
\text { by vi- } \\
\text { bration }\end{array}$ & 0.4 & $\begin{array}{l}0.16 \mathrm{~m} / \mathrm{d} \\
\text { (pore water } \\
\text { velocity) }\end{array}$ & $\begin{array}{l}\text { stain- } \\
\text { less } \\
\text { steel } \\
\text { mesh }\end{array}$ & $n / a$ & $\begin{array}{l}\text { peristal- } \\
\text { tic pump }\end{array}$ & natural water & $1 \mu \mathrm{g} / \mathrm{L}$ & $\mathrm{n} / \mathrm{a}$ & manual \\
\hline $\begin{array}{l}\text { Casas and } \\
\text { Bester (2015) }\end{array}$ & $\begin{array}{l}\text { saturated } \\
\text { flow } \\
\text { through }\end{array}$ & $\begin{array}{l}\text { diclofenac, } \\
\text { iohexol, } \\
\text { iomeprol, } \\
\text { iopromide, } \\
\text { propiconazole, } \\
\text { propranolol, } \\
\text { tebuconazole }\end{array}$ & glass & $\begin{array}{l}50 \\
\text { (filled } \\
\text { up to } \\
29 \text { ) }\end{array}$ & 2.5 & $\begin{array}{l}\text { artificial } \\
\text { sediment } \\
\text { (filter } \\
\text { sand/tech- } \\
\text { nical } \\
\text { quartz } \\
\text { sand) }\end{array}$ & $\mathrm{n} / \mathrm{a}$ & $\begin{array}{l}0.36 \\
\text { (from bro- } \\
\text { mide } \\
\text { tracer } \\
\text { test) }\end{array}$ & $\begin{array}{l}0.025-0.2 \\
\mathrm{~mL} / \mathrm{min}\end{array}$ & $\begin{array}{l}\text { glass-fi- } \\
\text { ber filter } \\
(1.2 \\
\mu \mathrm{m})\end{array}$ & $n / a$ & $\begin{array}{l}\text { piston } \\
\text { pump }\end{array}$ & $\begin{array}{l}\text { treated waste wa- } \\
\text { ter }\end{array}$ & $\begin{array}{l}0.022- \\
20.8 \mu \mathrm{g} / \mathrm{L}\end{array}$ & $\mathrm{n} / \mathrm{a}$ & $\begin{array}{l}\text { on-line } \\
\text { (HPLC-UV) \& } \\
\text { off-line } \\
\text { (HPLC- } \\
\text { MS/MS) }\end{array}$ \\
\hline $\begin{array}{l}\text { Chen et al. } \\
\text { (2011) }\end{array}$ & $\begin{array}{l}\text { saturated } \\
\text { flow } \\
\text { through }\end{array}$ & $\begin{array}{l}\text { ciprofloxacin, } \\
\text { sulfamethoxazole }\end{array}$ & $\begin{array}{l}\text { acrylic } \\
\text { cylinder }\end{array}$ & 10 & 2.5 & $\begin{array}{l}\text { artificial } \\
\text { sediment } \\
\text { (filter sand } \\
/ \text { technical } \\
\text { quartz } \\
\text { sand) }\end{array}$ & $\begin{array}{l}\text { wet, } \\
\text { stirring } \\
\text { and } \\
\text { tapping }\end{array}$ & 0.42 & $0.2 \mathrm{~cm} / \mathrm{min}$ & $\mathrm{n} / \mathrm{a}$ & $n / a$ & $\begin{array}{l}\text { peristal- } \\
\text { tic pump }\end{array}$ & $\begin{array}{l}\text { deionized water } \\
\text { with } \\
0.1 \mathrm{mM} \mathrm{KBr} \\
\text { pH } 9.5(\mathrm{NaOH})\end{array}$ & $\begin{array}{l}200 \mu \mathrm{\mu g} / \mathrm{L} \\
\text { sulfa- } \\
\text { methoxa- } \\
\text { zole, } 50 \\
\mu \mathrm{g} / \mathrm{L} \\
\text { ciproflox- } \\
\text { acin }\end{array}$ & $\begin{array}{l}\text { 1D- } \\
\text { CDE } \\
\text { coupled } \\
\text { with re- } \\
\text { action } \\
\text { terms }\end{array}$ & $\begin{array}{l}\text { fraction col- } \\
\text { lector }\end{array}$ \\
\hline $\begin{array}{l}\text { D'Alessio et } \\
\text { al. (2015) }\end{array}$ & $\begin{array}{l}\text { saturated } \\
\text { flow } \\
\text { through }\end{array}$ & $\begin{array}{l}17-\beta \text { estradiol, } \\
\text { caffeine, } \\
\text { carbamazepine, estrone, } \\
\text { gemfibrozil, } \\
\text { phenazone }\end{array}$ & $\begin{array}{l}\text { stainless } \\
\text { steel }\end{array}$ & 14.5 & 4.75 & $\begin{array}{l}\text { artificial } \\
\text { sediment } \\
\text { (filter sand } \\
/ \text { technical } \\
\text { quartz } \\
\text { sand) }\end{array}$ & $\mathrm{n} / \mathrm{a}$ & 0.41 & $0.2 \mathrm{~mL} / \mathrm{min}$ & $\begin{array}{l}\text { fine } \\
\text { stain- } \\
\text { less } \\
\text { steel } \\
\text { wire } \\
\text { cloth }\end{array}$ & $n / a$ & $\begin{array}{l}\text { peristal- } \\
\text { tic pump }\end{array}$ & $\begin{array}{l}\text { natural surface } \\
\text { water (aerobic \& } \\
\text { anaerobic) }\end{array}$ & $50 \mu \mathrm{g} / \mathrm{L}$ & $\mathrm{n} / \mathrm{a}$ & $\mathrm{n} / \mathrm{a}$ \\
\hline
\end{tabular}




\begin{tabular}{|c|c|c|c|c|c|c|c|c|c|c|c|c|c|c|c|c|}
\hline \multirow{2}{*}{ Citation } & \multirow{2}{*}{$\begin{array}{l}\text { Condi- } \\
\text { tions }\end{array}$} & \multirow{2}{*}{ Compounds } & \multicolumn{3}{|c|}{ Column } & \multicolumn{3}{|c|}{ Sediment } & \multirow{2}{*}{\begin{tabular}{|l} 
Velocities / \\
flowrates
\end{tabular}} & \multirow{2}{*}{$\begin{array}{l}\text { Filter } \\
\text { layers }\end{array}$} & \multicolumn{3}{|c|}{ Infrastructure / used materials } & \multirow{2}{*}{$\begin{array}{l}\text { Input } \\
\text { concen- } \\
\text { trations }\end{array}$} & \multirow{2}{*}{$\begin{array}{l}\text { Model- } \\
\text { ling }\end{array}$} & \multirow{2}{*}{$\begin{array}{l}\text { Sampling } \\
\text { methods }\end{array}$} \\
\hline & & & material & $\begin{array}{l}\text { length } \\
\text { [cm] }\end{array}$ & $\begin{array}{c}\text { diameter } \\
\text { [cm] }\end{array}$ & type & $\begin{array}{l}\text { instal- } \\
\text { lation }\end{array}$ & porosity & & & Tubes & Pump & Fluids & & & \\
\hline $\begin{array}{l}\text { Estrella et al. } \\
\text { (1993) }\end{array}$ & saturated & $\begin{array}{l}\text { 2,4-dichlorophenoxyace- } \\
\text { tic acid }(2,4-D)\end{array}$ & $\begin{array}{l}\text { n/a } \\
\text { (“chroma- } \\
\text { tography } \\
\text { column” } \\
\rightarrow \text { proba- } \\
\text { bly glass) }\end{array}$ & 5 & 2.5 & natural soil & $\mathrm{n} / \mathrm{a}$ & $\mathrm{n} / \mathrm{a}$ & $\begin{array}{l}4.7-6.2 \\
\mathrm{~cm} / \mathrm{h}\end{array}$ & $\mathrm{n} / \mathrm{a}$ & $n / a$ & $\mathrm{n} / \mathrm{a}$ & $\begin{array}{l}\text { artificial } \\
\text { (ground)water } \\
\left(\mathrm{CaCl}_{2}\right)\end{array}$ & $100 \mathrm{mg} / \mathrm{L}$ & $\begin{array}{l}\text { CDE, } \\
\text { incl. first } \\
\text { order } \\
\text { degra- } \\
\text { dation } \\
\text { (Van } \\
\text { Genuch } \\
\text { ten and } \\
\text { Wagen } \\
\text { et, } \\
1989 \text { ) }\end{array}$ & $\begin{array}{l}\text { fraction col- } \\
\text { lector }\end{array}$ \\
\hline $\begin{array}{l}\text { Greenhagen } \\
\text { et al. (2014) }\end{array}$ & $\begin{array}{l}\text { saturated } \\
\text { flow } \\
\text { through }\end{array}$ & $\begin{array}{l}\text { acetaminophen, } \\
\text { caffeine, } \\
\text { methamphetamine }\end{array}$ & PVC & 30 & 30 & $\begin{array}{l}\text { natural } \\
\text { soil, artifi- } \\
\text { cial sedi- } \\
\text { ment (filter } \\
\text { sand / } \\
\text { technical } \\
\text { quartz } \\
\text { sand) }\end{array}$ & $\begin{array}{l}\text { undis- } \\
\text { turbed } \\
\text { (soil); } \\
\text { n/a for } \\
\text { sand }\end{array}$ & $\begin{array}{l}0.28 \text { (un- } \\
\text { dis- } \\
\text { turbed) }\end{array}$ & $600 \mathrm{~mL} / \mathrm{d}$ & $\begin{array}{l}\text { quartz } \\
\text { filter } \\
\text { sand / } \\
\text { gravel, } \\
\text { alumi- } \\
\text { num } \\
\text { screen }\end{array}$ & $\begin{array}{l}\text { black } \\
\text { Tygon, } \\
\text { PVC } \\
\text { pump } \\
\text { tubing, } \\
\text { Vinyl, } \\
\text { brass } \\
\text { (com- } \\
\text { pression } \\
\text { fittings), } \\
\text { Polysul- } \\
\text { fone } \\
\text { (tees) }\end{array}$ & $\begin{array}{l}\text { peristal- } \\
\text { tic pump }\end{array}$ & $\begin{array}{l}\text { natural groundwa- } \\
\text { ter }\end{array}$ & $\begin{array}{l}1000 \\
\mathrm{mg} / \mathrm{L} \text { ac- } \\
\text { etamino- } \\
\text { phen, 250 } \\
\mathrm{mg} / \mathrm{L} \text { caf- } \\
\text { feine, } 100 \\
\mathrm{mg} / \mathrm{L} \\
\text { metham- } \\
\text { pheta- } \\
\text { mine }\end{array}$ & $\begin{array}{l}\text { CDE, } \\
\text { incl. first } \\
\text { order } \\
\text { degra- } \\
\text { dation } \\
\text { (Van } \\
\text { Genuch } \\
\text { ten and } \\
\text { Wagen } \\
\text { et, } \\
\text { 1989) }\end{array}$ & manual \\
\hline $\begin{array}{l}\text { Gruenheid et } \\
\text { al. (2008) }\end{array}$ & $\begin{array}{l}\text { saturated } \\
\text { flow } \\
\text { through }\end{array}$ & $\begin{array}{l}\text { iopromide, } \\
\text { sulfamethoxazole, } \\
\text { naphthalenedisulfonic } \\
\text { acid (each isomer) }\end{array}$ & plexiglas & 50 & 14 & $\begin{array}{l}\text { artificial } \\
\text { sediment } \\
\text { (filter sand } \\
\text { / technical } \\
\text { quartz } \\
\text { sand) }\end{array}$ & $\mathrm{n} / \mathrm{a}$ & $\mathrm{n} / \mathrm{a}$ & $\begin{array}{l}8.3 \mathrm{~cm} / \mathrm{d} \\
\text { (pore water } \\
\text { velocity) }\end{array}$ & $\begin{array}{l}\text { quartz } \\
\text { filter } \\
\text { sand / } \\
\text { gravel }\end{array}$ & $n / a$ & $\begin{array}{l}\text { peristal- } \\
\text { tic pump }\end{array}$ & $\begin{array}{l}\text { natural surface } \\
\text { water }\end{array}$ & $\begin{array}{l}10 \mu \mathrm{g} / \mathrm{L} \\
\text { iopromide } \\
, \\
2.5 \mu \mathrm{g} / \mathrm{L} \\
\text { sulfa- } \\
\text { methoxa- } \\
\text { zole, } \\
2.5 \mu \mathrm{g} / \mathrm{L} \\
\text { each iso- } \\
\text { mer of } \\
\text { naphtha- } \\
\text { lenedisul- } \\
\text { fonic acid }\end{array}$ & $\mathrm{n} / \mathrm{a}$ & $\mathrm{n} / \mathrm{a}$ \\
\hline $\begin{array}{l}\text { Hebig et al. } \\
\text { (submitted) }\end{array}$ & $\begin{array}{l}\text { saturated } \\
\text { flow } \\
\text { through }\end{array}$ & $\begin{array}{l}\text { caffeine, } \\
\text { carbamazepine, } \\
\text { gemfibrozil, } \\
\text { ibuprofen, } \\
\text { naproxen, } \\
\text { sulfamethoxazole }\end{array}$ & \begin{tabular}{|l} 
acrylic \\
glass
\end{tabular} & $\begin{array}{l}41.0- \\
41.2\end{array}$ & 7.6 & $\begin{array}{l}\text { one natu- } \\
\text { ral sedi- } \\
\text { ment, two } \\
\text { artificial } \\
\text { sands (or- } \\
\text { ganic car- } \\
\text { bon en- } \\
\text { riched } \\
\text { sand, iron } \\
\text { coated } \\
\text { sand) }\end{array}$ & $\begin{array}{l}\text { dry, } \\
\text { layers } \\
\text { of } 1-2 \\
\text { cm, } \\
\text { tamper- } \\
\text { ing }\end{array}$ & $\begin{array}{l}0.27- \\
0.33\end{array}$ & $\begin{array}{l}9.0-9.7 \\
\mathrm{~cm} / \mathrm{d} \\
\text { (pore water } \\
\text { velocity } \\
\text { from tracer } \\
\text { test) }\end{array}$ & $\begin{array}{l}\text { tech- } \\
\text { nical } \\
\text { quartz } \\
\text { sand ( } 2 \\
\mathrm{~cm} \text { top } \\
\text { and bot- } \\
\text { tom); } \\
\text { two } \\
\text { gauzes }\end{array}$ & Teflon & $\begin{array}{l}\text { peristal- } \\
\text { tic pump }\end{array}$ & $\begin{array}{l}\text { synthetic ground- } \\
\text { water }\end{array}$ & $\begin{array}{l}1 \mu \mathrm{g} / \mathrm{L} \text { of } \\
\text { each } \\
\text { com- } \\
\text { pound }\end{array}$ & $\begin{array}{l}\text { CXTFIT } \\
\text { (Toride } \\
\text { et al., } \\
1999 \text { ) }\end{array}$ & $\begin{array}{l}\text { sampling by } \\
\text { hand (bot- } \\
\text { tles) }\end{array}$ \\
\hline
\end{tabular}




\begin{tabular}{|c|c|c|c|c|c|c|c|c|c|c|c|c|c|c|c|c|}
\hline \multirow{2}{*}{ Citation } & \multirow{2}{*}{$\begin{array}{l}\text { Condi- } \\
\text { tions }\end{array}$} & \multirow{2}{*}{ Compounds } & \multicolumn{3}{|c|}{ Column } & \multicolumn{3}{|c|}{ Sediment } & \multirow{2}{*}{\begin{tabular}{|l} 
Velocities / \\
flowrates
\end{tabular}} & \multirow{2}{*}{$\begin{array}{l}\text { Filter } \\
\text { layers }\end{array}$} & \multicolumn{3}{|c|}{ Infrastructure / used materials } & \multirow{2}{*}{$\begin{array}{l}\text { Input } \\
\text { concen- } \\
\text { trations }\end{array}$} & \multirow{2}{*}{$\begin{array}{l}\text { Model- } \\
\text { ling }\end{array}$} & \multirow{2}{*}{$\begin{array}{l}\text { Sampling } \\
\text { methods }\end{array}$} \\
\hline & & & material & $\begin{array}{l}\text { length } \\
\text { [cm] }\end{array}$ & $\begin{array}{c}\text { diameter } \\
\text { [cm] }\end{array}$ & type & $\begin{array}{l}\text { instal- } \\
\text { lation }\end{array}$ & porosity & & & Tubes & Pump & Fluids & & & \\
\hline $\begin{array}{l}\text { Jia et al. } \\
\text { (2007) }\end{array}$ & $\begin{array}{l}\text { saturated } \\
\text { flow } \\
\text { through }\end{array}$ & benzotriazole & $\begin{array}{l}\text { plastipak } \\
\text { tube }\end{array}$ & 12 & 2.6 & \begin{tabular}{|l} 
natural \\
soil, artifi- \\
cial sedi- \\
ment \\
(zerovalent \\
iron $\mathrm{Fe}(0))$
\end{tabular} & $\mathrm{n} / \mathrm{a}$ & $\begin{array}{l}0.37 \text { (soil) } \\
0.41 / \\
0.44 \\
(\mathrm{Fe}(0))\end{array}$ & $\begin{array}{l}\text { different } \\
\text { flow rates } \\
\text { (from } 20 \text { to } \\
27.1 \mathrm{~mL} / \mathrm{h} \text { ) }\end{array}$ & $\begin{array}{l}\text { glass } \\
\text { wool }\end{array}$ & tygon & $\begin{array}{l}\text { peristal- } \\
\text { tic pump }\end{array}$ & distilled water & $10 \mathrm{mg} / \mathrm{L}$ & $\begin{array}{l}\text { PHREE } \\
\text { QC-2 } \\
\text { (Parkhu } \\
\text { rst and } \\
\text { Appelo, } \\
\text { 1999) }\end{array}$ & $\begin{array}{l}\text { flow-through } \\
\text { bottles }\end{array}$ \\
\hline $\begin{array}{l}\text { Ke et al. } \\
\text { (2012) }\end{array}$ & $\begin{array}{l}\text { saturated } \\
\text { flow } \\
\text { through }\end{array}$ & $\begin{array}{l}\text { 17a-ethynylestradiol, } \\
17 \beta \text {-estradiol, } \\
\text { bisphenol A, } \\
\text { ibuprofen, } \\
\text { naproxen }\end{array}$ & $n / a$ & $\begin{array}{l}8 \times 100 \\
\text { (series } \\
\text { connec- } \\
\text { tion) }\end{array}$ & 10 & $\begin{array}{l}\text { natural } \\
\text { sediment }\end{array}$ & $\mathrm{n} / \mathrm{a}$ & 0.44 & $22.7 \mathrm{~cm} / \mathrm{d}$ & $\mathrm{n} / \mathrm{a}$ & $\begin{array}{l}\text { Teflon } \\
\text { (PTFE), } \\
\text { stainless } \\
\text { steel }\end{array}$ & $\mathrm{n} / \mathrm{a}$ & $\begin{array}{l}\text { treated waste wa- } \\
\text { ter }\end{array}$ & $\begin{array}{l}\sim 100 \\
\mu \mathrm{g} / \mathrm{L}\end{array}$ & $\mathrm{n} / \mathrm{a}$ & $\begin{array}{l}\text { glass syringe } \\
\& \text { three-way } \\
\text { valves }\end{array}$ \\
\hline $\begin{array}{l}\text { Lorphensri et } \\
\text { al. (2007) }\end{array}$ & $\begin{array}{l}\text { saturated } \\
\text { flow } \\
\text { through }\end{array}$ & $\begin{array}{l}\text { 17a-ethynylestradiol, } \\
\text { acetaminophen, } \\
\text { nalidixic acid }\end{array}$ & $\begin{array}{l}\text { glass } \\
\text { (borosili- } \\
\text { cat) }\end{array}$ & 15 & 2.5 & \begin{tabular}{|l} 
artificial \\
sediment \\
(alumina \\
$\mathrm{Al}_{2} \mathrm{O}_{3}$, sil- \\
ica gel) \\
and aqui- \\
fer sand
\end{tabular} & $\mathrm{n} / \mathrm{a}$ & $\begin{array}{l}0.38- \\
0.52\end{array}$ & $\begin{array}{l}10.5-18.8 \\
\mathrm{~cm} / \mathrm{h}\end{array}$ & $\begin{array}{l}\text { HDPE } \\
(20 \mu \mathrm{m} \\
\text { porous } \\
\text { bed } \\
\text { support })\end{array}$ & $n / a$ & $\begin{array}{l}\text { peristal- } \\
\text { tic pump }\end{array}$ & $\begin{array}{l}\text { artificial } \\
\text { (ground)water } \\
\left(\mathrm{CaCl}_{2}\right)\end{array}$ & $10 \mathrm{mg} / \mathrm{L}$ & $\begin{array}{l}\text { CXT- } \\
\text { FIT, } \\
\text { CDE } \\
\text { (Toride } \\
\text { et al., } \\
\text { 1995), } \\
\text { UFBTC( } \\
\text { Universi } \\
\text { ty of } \\
\text { Florida, } \\
\text { 1989) }\end{array}$ & $\begin{array}{l}\text { fraction col- } \\
\text { lector, inline- } \\
\text { UV spectro- } \\
\text { photometer }\end{array}$ \\
\hline $\begin{array}{l}\text { Massmann et } \\
\text { al. (2008) }\end{array}$ & $\begin{array}{l}\text { down- } \\
\text { ward sat- } \\
\text { urated } \\
\text { flow- } \\
\text { through }\end{array}$ & $\begin{array}{l}\text { phenazone-type phar- } \\
\text { maceuticals and metab- } \\
\text { olites: } \\
\text { 1-acetyl-1-methyl-2-di- } \\
\text { methyloxamoyl-2-phe- } \\
\text { nylhydrazide, } \\
\text { 1-acetyl-1-methyl-2-phe- } \\
\text { nylhydrazide, } \\
\text { 1,5-dimethyl-1,2-dehy- } \\
\text { dro-3-pyrazolone, } \\
\text { 4-acetylaminoantipyrin, } \\
\text { 4-formylaminoantipyrin, } \\
\text { 4-(2-methylethyl)-1,5-di- } \\
\text { methyl-1,2-dehydro-3- } \\
\text { pyrazole, } \\
\text { dimethylaminophena- } \\
\text { zone, } \\
\text { phenazone, } \\
\text { propyphenazone, }\end{array}$ & $n / a$ & $\mathrm{n} / \mathrm{a}$ & $\mathrm{n} / \mathrm{a}$ & $\begin{array}{l}\text { undis- } \\
\text { turbed } \\
\text { core of } \\
\text { sediments } \\
\text { from the } \\
\text { Lake } \\
\text { Wannsee } \\
\text { bed }\end{array}$ & $\begin{array}{l}\text { undis- } \\
\text { turbed }\end{array}$ & $\mathrm{n} / \mathrm{a}$ & $\begin{array}{l}6^{*} 10^{-6} \mathrm{~m} / \mathrm{s} \\
=0.52 \mathrm{~m} / \mathrm{d} \\
\text { (pore veloc- } \\
\text { ity) }\end{array}$ & $\mathrm{n} / \mathrm{a}$ & $n / a$ & $\begin{array}{l}\text { pulsating } \\
\text { pump at } \\
\text { the bot- } \\
\text { tom of } \\
\text { the col- } \\
\text { umn }\end{array}$ & $\begin{array}{l}\text { natural surface } \\
\text { (lake) water }\end{array}$ & $\begin{array}{l}0.03- \\
0.53 \mu \mathrm{g} / \mathrm{L} \\
\text { (median } \\
\text { Lake } \\
\text { Wannsee) }\end{array}$ & $\mathrm{n} / \mathrm{a}$ & $\begin{array}{l}\text { redox-condi- } \\
\text { tions: oxygen } \\
\text { minisensors } \\
\text { in the sedi- } \\
\text { ment }\end{array}$ \\
\hline $\begin{array}{l}\text { Mersmann et } \\
\text { al. (2002) }\end{array}$ & $\begin{array}{l}\text { saturated } \\
\text { flow } \\
\text { through }\end{array}$ & $\begin{array}{l}\text { carbamazepine, } \\
\text { clofibric acid, } \\
\text { diclofenac, } \\
\text { ibuprofen, } \\
\text { propyphenazone }\end{array}$ & $\begin{array}{l}\text { stainless } \\
\text { steel }\end{array}$ & 35 & 13.59 & $\begin{array}{l}\text { natural } \\
\text { sediment }\end{array}$ & $\mathrm{n} / \mathrm{a}$ & $\begin{array}{l}0.27- \\
0.36\end{array}$ & $\begin{array}{l}0.33-0.36 \\
\mathrm{~m} / \mathrm{d} \text { (mean } \\
\text { pore veloc- } \\
\text { ity) }\end{array}$ & $\mathrm{n} / \mathrm{a}$ & $\begin{array}{l}\text { Teflon, } \\
\text { glass, } \\
\text { polyeth- } \\
\text { ylene, } \\
\text { stainless } \\
\text { steel }\end{array}$ & $\begin{array}{l}\text { peristal- } \\
\text { tic pump }\end{array}$ & $\begin{array}{l}\text { natural groundwa- } \\
\text { ter }\end{array}$ & $\begin{array}{l}10 \mu \mathrm{g} / \mathrm{L} \\
\text { each } \\
\text { com- } \\
\text { pound }\end{array}$ & $\mathrm{n} / \mathrm{a}$ & $\begin{array}{l}\text { flowthrough } \\
\text { cells, fraction } \\
\text { collector }\end{array}$ \\
\hline
\end{tabular}




\begin{tabular}{|c|c|c|c|c|c|c|c|c|c|c|c|c|c|c|c|c|}
\hline \multirow{2}{*}{ Citation } & \multirow{2}{*}{$\begin{array}{l}\text { Condi- } \\
\text { tions }\end{array}$} & \multirow{2}{*}{ Compounds } & \multicolumn{3}{|c|}{ Column } & \multicolumn{3}{|c|}{ Sediment } & \multirow{2}{*}{$\begin{array}{l}\text { Velocities / } \\
\text { flowrates }\end{array}$} & \multirow{2}{*}{$\begin{array}{l}\text { Filter } \\
\text { layers }\end{array}$} & \multicolumn{3}{|c|}{ Infrastructure / used materials } & \multirow{2}{*}{$\begin{array}{l}\text { Input } \\
\text { concen- } \\
\text { trations }\end{array}$} & \multirow{2}{*}{$\begin{array}{l}\text { Model- } \\
\text { ling }\end{array}$} & \multirow{2}{*}{$\begin{array}{l}\text { Sampling } \\
\text { methods }\end{array}$} \\
\hline & & & material & $\begin{array}{l}\text { length } \\
{[\mathrm{cm}]}\end{array}$ & $\begin{array}{c}\text { diameter } \\
\text { [cm] }\end{array}$ & type & $\begin{array}{l}\text { instal- } \\
\text { lation } \\
\end{array}$ & porosity & & & Tubes & Pump & Fluids & & & \\
\hline $\begin{array}{l}\text { Müller et al. } \\
(2013)\end{array}$ & $\begin{array}{l}\text { saturated } \\
\text { flow } \\
\text { through }\end{array}$ & $\begin{array}{l}\text { carbamazepine, } \\
\text { primidone, } \\
\text { sulfamethoxazole }\end{array}$ & $n / a$ & 35 & 13.5 & $\begin{array}{l}\text { natural } \\
\text { sediment } \\
\text { (un- and } \\
\text { heat pre- } \\
\text { treated) }\end{array}$ & $\mathrm{n} / \mathrm{a}$ & 0.35 & $\begin{array}{l}1.20-1.56 \\
\mathrm{~cm} / \mathrm{h} \text { (pore } \\
\text { velocity) }\end{array}$ & $\mathrm{n} / \mathrm{a}$ & $\mathrm{n} / \mathrm{a}$ & $\begin{array}{l}\text { peristal- } \\
\text { tic pump }\end{array}$ & $\begin{array}{l}\text { natural surface } \\
\text { water (ozonated } \\
\text { and not-ozonated) }\end{array}$ & $\begin{array}{l}0.73-0.8 \\
\mu \mathrm{g} / \mathrm{L} \\
\text { primidone } \\
, \\
0.80 \mu \mathrm{g} / \mathrm{L} \\
\text { carbam- } \\
\text { azepine, } \\
0.87- \\
0.90 \mu \mathrm{h} / \mathrm{L} \\
\text { sulfa- } \\
\text { methoxa- } \\
\text { zole }\end{array}$ & $\begin{array}{l}\text { CXT- } \\
\text { FIT, } \\
\text { CDE } \\
\text { (Toride } \\
\text { et al., } \\
1999 \text { ) }\end{array}$ & $\begin{array}{l}\text { flowthrough } \\
\text { cells, fraction } \\
\text { collector }\end{array}$ \\
\hline $\begin{array}{l}\text { Patterson et } \\
\text { al. (2010) }\end{array}$ & $\begin{array}{l}\text { saturated } \\
\text { flow } \\
\text { through }\end{array}$ & $\begin{array}{l}\text { 17a-ethynylestradiol, } \\
17 \beta \text {-estradiol, } \\
\text { bisphenol A, } \\
\text { carbamazepine, } \\
\text { iodipamide, } \\
\text { iohexol, } \\
\mathrm{N} \text {-nitrosomorpholine, } \\
\mathrm{N} \text {-nitrosodimethylamine } \\
\text { oxazepam }\end{array}$ & $\begin{array}{l}\text { stainless } \\
\text { steel }\end{array}$ & 200 & 14.5 & $\begin{array}{l}\text { anaerobic } \\
\text { natural } \\
\text { sediment }\end{array}$ & $\mathrm{n} / \mathrm{a}$ & $\begin{array}{l}0.42 \text { (bro- } \\
\text { mide } \\
\text { tracer } \\
\text { test) }\end{array}$ & $0.052 \mathrm{~m} / \mathrm{d}$ & $\begin{array}{l}\text { stain- } \\
\text { less } \\
\text { steel } \\
\text { mesh } \\
\text { and } \\
\text { grate }\end{array}$ & $\mathrm{n} / \mathrm{a}$ & $\begin{array}{l}\text { peristal- } \\
\text { tic pump }\end{array}$ & $\begin{array}{l}\text { treated waste wa- } \\
\text { ter }\end{array}$ & $\begin{array}{l}130-700 \\
\mu \mathrm{g} / \mathrm{L}\end{array}$ & $\begin{array}{l}\text { fitting } \\
\text { using } \\
\text { Origin } \circledast \\
\text { v7 } \\
\text { (Microc } \\
\text { al } \\
\text { Softwar } \\
\text { e Inc., } \\
\text { 1995) }\end{array}$ & $\begin{array}{l}\text { hypodermic } \\
\text { syringe }\end{array}$ \\
\hline $\begin{array}{l}\text { Patterson et } \\
\text { al. (2011) }\end{array}$ & $\begin{array}{l}\text { saturated } \\
\text { flow } \\
\text { through }\end{array}$ & $\begin{array}{l}\text { 17a-ethynylestradiol, } \\
17 \beta \text {-estradiol, } \\
\text { bisphenol } \mathrm{A} \text {, } \\
\text { carbamazepine, } \\
\text { iodipamide, } \\
\text { iohexol, } \\
\mathrm{N} \text {-nitrosomorpholine, } \\
\mathrm{N} \text {-nitrosodimethylamine } \\
\text { oxazepam }\end{array}$ & $\begin{array}{l}\text { stainless } \\
\text { steel }\end{array}$ & 200 & 14.5 & $\begin{array}{l}\text { aerobic } \\
\text { natural } \\
\text { sediment }\end{array}$ & $\begin{array}{l}\text { wet } \\
\text { (satu- } \\
\text { rated) }\end{array}$ & $\begin{array}{l}0.46 \text { (bro- } \\
\text { mide } \\
\text { tracer } \\
\text { test) }\end{array}$ & $0.047 \mathrm{~m} / \mathrm{d}$ & $\begin{array}{l}\text { stain- } \\
\text { less } \\
\text { steel } \\
\text { mesh } \\
\text { and } \\
\text { grate, } \\
\text { silicone } \\
\text { polymer } \\
\text { mat }\end{array}$ & $\mathrm{n} / \mathrm{a}$ & $\begin{array}{l}\text { peristal- } \\
\text { tic pump }\end{array}$ & $\begin{array}{l}\text { treated waste wa- } \\
\text { ter }\end{array}$ & $\begin{array}{l}130-700 \\
\mu \mathrm{g} / \mathrm{L}\end{array}$ & $\begin{array}{l}\text { fitting } \\
\text { to CDE } \\
\text { using } \\
\text { Origin® } \\
\text { v7 } \\
\text { (Microc } \\
\text { al } \\
\text { Softwar } \\
\text { e Inc., } \\
\text { 1995) }\end{array}$ & $\begin{array}{l}\text { hypodermic } \\
\text { syringe }\end{array}$ \\
\hline $\begin{array}{l}\text { Preuss et al. } \\
(2001)\end{array}$ & $\begin{array}{l}\text { (proba- } \\
\text { bly) satu- } \\
\text { rated up- } \\
\text { ward and } \\
\text { down- } \\
\text { ward flow } \\
\text { through } \\
\text { (various } \\
\text { experi- } \\
\text { ments) }\end{array}$ & $\begin{array}{l}\text { bezafibrat, diclofenac, } \\
\text { carbamazepine, gemfib- } \\
\text { rozil, clofibric acid }\end{array}$ & glass & 80 & 20 & $\begin{array}{l}\text { natural } \\
\text { sediment }\end{array}$ & $\mathrm{n} / \mathrm{a}$ & $\mathrm{n} / \mathrm{a}$ & $\begin{array}{l}5 \mathrm{~cm} / \mathrm{h} \\
\text { (Darcy ve- } \\
\text { locity) }\end{array}$ & $\mathrm{n} / \mathrm{a}$ & $\mathrm{n} / \mathrm{a}$ & $\mathrm{n} / \mathrm{a}$ & $\begin{array}{l}\text { natural groundwa- } \\
\text { ter (aerobic \& an- } \\
\text { aerobic), natural } \\
\text { surface water }\end{array}$ & $\begin{array}{l}100 \mu \mathrm{g} / \mathrm{L} \\
\text { each } \\
\text { com- } \\
\text { pound }\end{array}$ & $\mathrm{n} / \mathrm{a}$ & $\mathrm{n} / \mathrm{a}$ \\
\hline
\end{tabular}




\begin{tabular}{|c|c|c|c|c|c|c|c|c|c|c|c|c|c|c|c|c|}
\hline \multirow{2}{*}{ Citation } & \multirow{2}{*}{$\begin{array}{l}\text { Condi- } \\
\text { tions }\end{array}$} & \multirow{2}{*}{ Compounds } & \multicolumn{3}{|c|}{ Column } & \multicolumn{3}{|c|}{ Sediment } & \multirow{2}{*}{$\begin{array}{l}\text { Velocities / } \\
\text { flowrates }\end{array}$} & \multirow{2}{*}{$\begin{array}{l}\text { Filter } \\
\text { layers }\end{array}$} & \multicolumn{3}{|c|}{ Infrastructure / used materials } & \multirow{2}{*}{$\begin{array}{l}\text { Input } \\
\text { concen- } \\
\text { trations }\end{array}$} & \multirow{2}{*}{$\begin{array}{l}\text { Model- } \\
\text { ling }\end{array}$} & \multirow{2}{*}{$\begin{array}{l}\text { Sampling } \\
\text { methods }\end{array}$} \\
\hline & & & material & $\begin{array}{l}\text { length } \\
\text { [cm] }\end{array}$ & $\begin{array}{c}\text { diameter } \\
{[\mathrm{cm}]}\end{array}$ & type & $\begin{array}{l}\text { instal- } \\
\text { lation }\end{array}$ & porosity & & & Tubes & Pump & Fluids & & & \\
\hline $\begin{array}{l}\text { Rauch- } \\
\text { Williams et al. } \\
\text { (2010) }\end{array}$ & $\begin{array}{l}\text { saturated } \\
\text { flow } \\
\text { through }\end{array}$ & $\begin{array}{l}\text { carbamazepine, } \\
\text { diclofenac sodium, } \\
\text { gemfibrozil, } \\
\text { ibuprofen, } \\
\text { ketoprofen, } \\
\text { naproxen, } \\
\text { phenacetine, } \\
\text { primidone, } \\
\text { propyphenazone, } \\
\text { tris(2-chloroethyl)phos- } \\
\text { phate }\end{array}$ & \begin{tabular}{|l} 
plexi- \\
glass
\end{tabular} & $\begin{array}{l}4 \times 100 \\
\text { (in se- } \\
\text { ries } \\
\text { connec- } \\
\text { tion) }\end{array}$ & 15 & \begin{tabular}{|l} 
natural \\
sediment
\end{tabular} & $\mathrm{n} / \mathrm{a}$ & $\mathrm{n} / \mathrm{a}$ & $\begin{array}{l}0.065 \mathrm{~m} / \mathrm{d} \\
\text { (loading } \\
\text { rate) }\end{array}$ & $\mathrm{n} / \mathrm{a}$ & $n / a$ & $\mathrm{n} / \mathrm{a}$ & $\begin{array}{l}\text { treated waste wa- } \\
\text { ter }\end{array}$ & \begin{tabular}{|l|}
$23-1,503$ \\
$\mathrm{ng} / \mathrm{L}$
\end{tabular} & $\begin{array}{l}\text { CXT- } \\
\text { FIT,CD } \\
\text { E } \\
\text { (Toride } \\
\text { et al., } \\
1995 \text { ) }\end{array}$ & $\mathrm{n} / \mathrm{a}$ \\
\hline $\begin{array}{l}\text { Schaffer et al. } \\
(2012 b)\end{array}$ & $\begin{array}{l}\text { saturated } \\
\text { flow } \\
\text { through }\end{array}$ & $\begin{array}{l}\text { atenolol, } \\
\text { metoprolol }\end{array}$ & $\begin{array}{l}\text { stainless } \\
\text { steel }\end{array}$ & 25 & 3.4 & $\begin{array}{l}\text { natural } \\
\text { sediment }\end{array}$ & $\mathrm{n} / \mathrm{a}$ & $\begin{array}{l}0.29- \\
0.38 \\
\text { (chloride } \\
\text { tracer } \\
\text { test) }\end{array}$ & $\begin{array}{l}0.62-0.75 \\
\mathrm{~m} / \mathrm{d} \text { (Darcy } \\
\text { velocity) } \\
1.91-2.43 \\
\mathrm{~m} / \mathrm{d} \text { (pore } \\
\text { velocity) }\end{array}$ & $\mathrm{n} / \mathrm{a}$ & $n / a$ & $\begin{array}{l}\text { piston } \\
\text { pump }\end{array}$ & tap water & $\begin{array}{l}1- \\
30,000 \\
\mu \mathrm{g} / \mathrm{L} \\
\text { atenolol } \\
500 \mu \mathrm{g} / \mathrm{L} \\
\text { metopro- } \\
\text { lol }\end{array}$ & $\begin{array}{l}\text { CXT- } \\
\text { FIT, } \\
\text { CDE } \\
\text { (Toride } \\
\text { et al., } \\
1995 \text { ) }\end{array}$ & $\begin{array}{l}\text { fraction col- } \\
\text { lector }\end{array}$ \\
\hline $\begin{array}{l}\text { Schaffer et al. } \\
\text { (2012a) }\end{array}$ & $\begin{array}{l}\text { saturated } \\
\text { flow } \\
\text { through }\end{array}$ & $\begin{array}{l}\text { atenolol, } \\
\text { carbamazepine, } \\
\text { cetirizine, } \\
\text { diazepam, } \\
\text { naproxen, } \\
\text { phenobarbital, } \\
\text { primidone, } \\
\text { trimethoprim }\end{array}$ & $\begin{array}{l}\text { stainless } \\
\text { steel }\end{array}$ & 25 & 3.4 & $\begin{array}{l}\text { natural } \\
\text { sediment }\end{array}$ & $\mathrm{n} / \mathrm{a}$ & $\begin{array}{l}0.34 \\
\text { (chloride } \\
\text { tracer } \\
\text { test) }\end{array}$ & $\begin{array}{l}1.28 \mathrm{~m} / \mathrm{d} \\
\text { (Darcv ve- } \\
\text { locity), } \\
3.77 \mathrm{~m} / \mathrm{d} \\
\text { (pore veloc- } \\
\text { ity) }\end{array}$ & $\mathrm{n} / \mathrm{a}$ & $n / a$ & $\mathrm{n} / \mathrm{a}$ & $\begin{array}{l}\text { natural surface } \\
\text { water }\end{array}$ & $500 \mu \mathrm{g} / \mathrm{L}$ & $\begin{array}{l}\text { fitting to } \\
\text { CDE }\end{array}$ & $\mathrm{n} / \mathrm{a}$ \\
\hline $\begin{array}{l}\text { Scheytt et al. } \\
\text { (1998) }\end{array}$ & $\begin{array}{l}\text { saturated } \\
\text { flow } \\
\text { through }\end{array}$ & clofibric acid & \begin{tabular}{|l} 
stainless \\
steel \\
(personal \\
commu- \\
nication)
\end{tabular} & 30 & 9 & $\begin{array}{l}\text { natural } \\
\text { sediment }\end{array}$ & $\mathrm{n} / \mathrm{a}$ & 0.34 & $\begin{array}{l}0.26 \mathrm{~m} / \mathrm{d} \\
\text { (Darcy ve- } \\
\text { locity) }\end{array}$ & $\mathrm{n} / \mathrm{a}$ & $n / a$ & $\begin{array}{l}\text { peristal- } \\
\text { tic pump }\end{array}$ & $\begin{array}{l}\text { natural groundwa- } \\
\text { ter (anaerobic) }\end{array}$ & $0.7 \mu \mathrm{g} / \mathrm{L}$ & $\mathrm{n} / \mathrm{a}$ & $\begin{array}{l}\text { flowthrough } \\
\text { cells, fraction } \\
\text { collector }\end{array}$ \\
\hline $\begin{array}{l}\text { Scheytt et al. } \\
(2004)\end{array}$ & $\begin{array}{l}\text { saturated } \\
\text { flow } \\
\text { through }\end{array}$ & $\begin{array}{l}\text { clofibric acid, } \\
\text { diclofenac, } \\
\text { propyphenazone }\end{array}$ & $\begin{array}{l}\text { stainless } \\
\text { steel }\end{array}$ & 35 & 13.6 & $\begin{array}{l}\text { natural } \\
\text { sediment }\end{array}$ & dry & 0.32 & $\begin{array}{l}0.30 \mathrm{~m} / \mathrm{d} \\
\text { (dominant } \\
\text { linear ve- } \\
\text { locity) }\end{array}$ & $\begin{array}{l}\text { glass } \\
(?) \\
\text { globes } \\
\text { and } \\
\text { gauze } \\
\text { net }\end{array}$ & $n / a$ & $\mathrm{n} / \mathrm{a}$ & $\begin{array}{l}\text { natural groundwa- } \\
\text { ter }\end{array}$ & $10 \mu \mathrm{g} / \mathrm{L}$ & $\begin{array}{l}\text { graph- } \\
\text { ical } \\
\text { analysis } \\
\text { of BTCs }\end{array}$ & $\begin{array}{l}\text { flowthrough } \\
\text { cells, fraction } \\
\text { collector }\end{array}$ \\
\hline
\end{tabular}




\begin{tabular}{|c|c|c|c|c|c|c|c|c|c|c|c|c|c|c|c|c|}
\hline \multirow{2}{*}{ Citation } & \multirow{2}{*}{$\begin{array}{l}\text { Condi- } \\
\text { tions }\end{array}$} & \multirow{2}{*}{ Compounds } & \multicolumn{3}{|c|}{ Column } & \multicolumn{3}{|c|}{ Sediment } & \multirow{2}{*}{$\begin{array}{l}\text { Velocities / } \\
\text { flowrates }\end{array}$} & \multirow{2}{*}{$\begin{array}{l}\text { Filter } \\
\text { layers }\end{array}$} & \multicolumn{3}{|c|}{ Infrastructure / used materials } & \multirow{2}{*}{$\begin{array}{l}\text { Input } \\
\text { concen- } \\
\text { trations }\end{array}$} & \multirow{2}{*}{$\begin{array}{l}\text { Model- } \\
\text { ling }\end{array}$} & \multirow{2}{*}{$\begin{array}{l}\text { Sampling } \\
\text { methods }\end{array}$} \\
\hline & & & material & $\begin{array}{l}\text { length } \\
\text { [cm] }\end{array}$ & $\begin{array}{c}\text { diameter } \\
{[\mathrm{cm}]}\end{array}$ & type & $\begin{array}{l}\text { instal- } \\
\text { lation }\end{array}$ & porosity & & & Tubes & Pump & Fluids & & & \\
\hline $\begin{array}{l}\text { Simon et al. } \\
(2000)\end{array}$ & $\begin{array}{l}\text { saturated } \\
\text { flow } \\
\text { through }\end{array}$ & p-cyanonitrobenzene & glass & 62 & 5 & \begin{tabular}{|l} 
natural \\
sediment
\end{tabular} & $\begin{array}{l}\text { wet } \\
\text { (satu- } \\
\text { rated) }\end{array}$ & $\begin{array}{l}0.47 \\
\text { (tracer } \\
\text { test) }\end{array}$ & $\begin{array}{l}2.5 \mathrm{~mL} / \mathrm{min} \\
(=16.25 \\
\mathrm{cm} / \mathrm{h}) \text { in the } \\
\text { tracer test; } \\
0.5 \mathrm{~mL} / \mathrm{min} \\
\text { during the } \\
\text { experiment }\end{array}$ & $\mathrm{n} / \mathrm{a}$ & $n / a$ & $\begin{array}{l}\text { Rabbit- } \\
\text { HP, } \\
\text { Rainin } \\
\text { Instru- } \\
\text { ment } \\
\text { Co. Inc } \\
\text { (mobile } \\
\text { phase), } \\
\text { syringe } \\
\text { pump } \\
\text { (injection } \\
\text { of com- } \\
\text { pound) }\end{array}$ & $\begin{array}{l}\text { ultrapure water } \\
\text { (air-saturated) }\end{array}$ & $25 \mu \mathrm{M}$ & $\begin{array}{l}\mathrm{CDE} \\
\text { model }\end{array}$ & $\begin{array}{l}\text { fraction col- } \\
\text { lector (tracer) } \\
\text { syringe } \\
\text { (compound) }\end{array}$ \\
\hline $\begin{array}{l}\text { Srivastava et } \\
\text { al. (2009) }\end{array}$ & $\begin{array}{l}\text { saturated } \\
\text { flow } \\
\text { through }\end{array}$ & $\begin{array}{l}\text { ormetoprim, } \\
\text { sulfadimethoxine }\end{array}$ & glass & $\begin{array}{l}10 \text { and } \\
4\end{array}$ & 5 & $\begin{array}{l}\text { natural } \\
\text { soil, artifi- } \\
\text { cial sedi- } \\
\text { ments (fil- } \\
\text { ter sand / } \\
\text { technical } \\
\text { quartz } \\
\text { sand) }\end{array}$ & dry & $\mathrm{n} / \mathrm{a}$ & $\begin{array}{l}0.27-0.48 \\
\mathrm{~cm} / \mathrm{min} \\
\text { (pore water } \\
\text { velocity) }\end{array}$ & $\begin{array}{l}\text { cheese- } \\
\text { cloth, } \\
\text { paper } \\
\text { filter, } \\
\text { Teflon } \\
\text { end cap }\end{array}$ & $\begin{array}{l}\text { polypro- } \\
\text { pylene, } \\
\text { silicone }\end{array}$ & $\begin{array}{l}\text { peristal- } \\
\text { tic pump }\end{array}$ & $\begin{array}{l}\text { artificial } \\
\text { (ground)water } \\
\left(\mathrm{CaCl}_{2}\right)\end{array}$ & $100 \mu \mathrm{g} / \mathrm{L}$ & $\begin{array}{l}\text { CXT- } \\
\text { FIT, } \\
\text { CDE } \\
\text { (Toride } \\
\text { et al., } \\
1999 \text { ) }\end{array}$ & $\begin{array}{l}\text { fraction col- } \\
\text { lector }\end{array}$ \\
\hline $\begin{array}{l}\text { Strauss et al. } \\
\text { (2011) }\end{array}$ & $\begin{array}{l}\text { saturated } \\
\text { flow } \\
\text { through }\end{array}$ & $\begin{array}{l}\text { sulfadimethoxine, sulfa- } \\
\text { methazine, } \\
\text { sulfamethoxazole }\end{array}$ & $\begin{array}{l}\text { stainless } \\
\text { steel }\end{array}$ & 30 & 5.2 & natural soil & $\begin{array}{l}\text { dry, } \\
\text { com- } \\
\text { paction } \\
\text { by tap- } \\
\text { ping } \\
\text { with } \\
\text { rubber } \\
\text { ham- } \\
\text { mer }\end{array}$ & $\begin{array}{l}0.38 \text { - } \\
0.40 \text { (po- } \\
\text { rosity as } \\
\text { water } \\
\text { content) }\end{array}$ & $\begin{array}{l}3.99-4.15 \\
\mathrm{~cm} / \mathrm{h} \\
\text { (Darcy ve- } \\
\text { locity) }\end{array}$ & $\begin{array}{l}\text { perfo- } \\
\text { rated } \\
\text { stain- } \\
\text { less } \\
\text { steel } \\
\text { plates, } \\
\text { glass fi- } \\
\text { ber filter }\end{array}$ & $\begin{array}{l}\text { Teflon } \\
\text { (PTFE), } \\
\text { pharmed }\end{array}$ & $\begin{array}{l}\text { peristal- } \\
\text { tic pump }\end{array}$ & $\begin{array}{l}\text { artificial } \\
\text { (ground)water } \\
\left(\mathrm{CaCl}_{2}\right) \text {, liquid ma- } \\
\text { nure }\end{array}$ & $500 \mu \mathrm{g} / \mathrm{L}$ & $\begin{array}{l}\text { HY- } \\
\text { DRUS- } \\
\text { 1D, } \\
\text { CDE } \\
\text { (Šimůn } \\
\text { ek and } \\
\text { Van } \\
\text { Genuch } \\
\text { ten, } \\
2008 \text { ) }\end{array}$ & $\begin{array}{l}\text { fraction col- } \\
\text { lector }\end{array}$ \\
\hline $\begin{array}{l}\text { Teijón et al. } \\
\text { (2014) }\end{array}$ & $\begin{array}{l}\text { saturated } \\
\text { flow } \\
\text { through }\end{array}$ & naproxen & $\begin{array}{l}\text { stainless } \\
\text { steel, the } \\
\text { internal } \\
\text { wall was } \\
\text { covered } \\
\text { with Tef- } \\
\text { lon }\end{array}$ & 10 & 1.6 & \begin{tabular}{|l} 
natural \\
sediment
\end{tabular} & $\begin{array}{l}\text { dry, } \\
\text { com- } \\
\text { paction } \\
\text { by vi- } \\
\text { bration }\end{array}$ & 0.27 & $\begin{array}{l}0.19-0.7 \\
\mathrm{~cm} / \mathrm{min} \\
\text { (pore water } \\
\text { velocity) }\end{array}$ & $\mathrm{n} / \mathrm{a}$ & $\begin{array}{l}\text { PTFE, } \\
\text { stainless } \\
\text { steel }\end{array}$ & $\begin{array}{l}\text { peristal- } \\
\text { tic pump }\end{array}$ & $\begin{array}{l}\text { artificial } \\
\text { (ground)water } \\
\left(\mathrm{CaCl}_{2}\right)\end{array}$ & $10 \mu \mathrm{g} / \mathrm{L}$ & $\begin{array}{l}\text { HY- } \\
\text { DRUS- } \\
\text { 1D, } \\
\text { CDE } \\
\text { (Šimůn } \\
\text { ek and } \\
\text { Van } \\
\text { Genuch } \\
\text { ten, } \\
2008 \text { ) }\end{array}$ & $\begin{array}{l}\text { fraction col- } \\
\text { lector }\end{array}$ \\
\hline
\end{tabular}




\begin{tabular}{|c|c|c|c|c|c|c|c|c|c|c|c|c|c|c|c|c|}
\hline \multirow{2}{*}{ Citation } & \multirow{2}{*}{$\begin{array}{l}\text { Condi- } \\
\text { tions }\end{array}$} & \multirow{2}{*}{ Compounds } & \multicolumn{3}{|c|}{ Column } & \multicolumn{3}{|c|}{ Sediment } & \multirow{2}{*}{$\begin{array}{l}\text { Velocities / } \\
\text { flowrates }\end{array}$} & \multirow{2}{*}{$\begin{array}{l}\text { Filter } \\
\text { layers }\end{array}$} & \multicolumn{3}{|c|}{ Infrastructure / used materials } & \multirow{2}{*}{\begin{tabular}{|c|} 
Input \\
concen- \\
trations
\end{tabular}} & \multirow{2}{*}{$\begin{array}{l}\text { Model- } \\
\text { ling }\end{array}$} & \multirow{2}{*}{$\begin{array}{l}\text { Sampling } \\
\text { methods }\end{array}$} \\
\hline & & & material & $\begin{array}{l}\text { length } \\
{[\mathrm{cm}]}\end{array}$ & $\begin{array}{c}\text { diameter } \\
{[\mathrm{cm}]}\end{array}$ & type & $\begin{array}{l}\text { instal- } \\
\text { lation }\end{array}$ & porosity & & & Tubes & Pump & Fluids & & & \\
\hline $\begin{array}{l}\text { Teijón et al. } \\
\text { (2014) }\end{array}$ & $\begin{array}{l}\text { saturated } \\
\text { flow } \\
\text { through }\end{array}$ & naproxen & \begin{tabular}{|l|} 
stainless \\
steel, the \\
internal \\
wall was \\
covered \\
with Tef- \\
lon
\end{tabular} & 5 & 2 & \begin{tabular}{|l} 
natural \\
sediment
\end{tabular} & $\begin{array}{l}\text { dry, } \\
\text { com- } \\
\text { paction } \\
\text { by vi- } \\
\text { bration }\end{array}$ & 0.25 & $\begin{array}{l}0.08 \\
\mathrm{~cm} / \mathrm{min}\end{array}$ & $\mathrm{n} / \mathrm{a}$ & \begin{tabular}{|l} 
PTFE, \\
stainless \\
steel
\end{tabular} & $\begin{array}{l}\text { peristal- } \\
\text { tic pump }\end{array}$ & $\begin{array}{l}\text { artificial } \\
\text { (ground)water } \\
\left(\mathrm{CaCl}_{2}\right)\end{array}$ & $10 \mu \mathrm{g} / \mathrm{L}$ & $\begin{array}{l}\text { HY- } \\
\text { DRUS- } \\
\text { 1D, } \\
\text { CDE } \\
\text { (Šimůn } \\
\text { ek and } \\
\text { Van } \\
\text { Genuch } \\
\text { ten, } \\
\text { 2008) }\end{array}$ & $\begin{array}{l}\text { fraction col- } \\
\text { lector }\end{array}$ \\
\hline
\end{tabular}




\begin{tabular}{|c|c|c|c|c|c|c|c|c|c|c|c|c|c|c|c|c|}
\hline \multirow[b]{2}{*}{ Citation } & \multirow{2}{*}{$\begin{array}{l}\text { Condi- } \\
\text { tions }\end{array}$} & \multirow[b]{2}{*}{ Compounds } & \multicolumn{3}{|c|}{ Column } & \multicolumn{3}{|c|}{ Sediment } & \multirow{2}{*}{$\begin{array}{l}\text { Veloci- } \\
\text { ties / } \\
\text { flowrates }\end{array}$} & \multirow{2}{*}{$\begin{array}{l}\text { Filter lay- } \\
\text { ers }\end{array}$} & \multicolumn{3}{|c|}{ Infrastructure / used materials } & \multirow{2}{*}{$\begin{array}{l}\text { Input } \\
\text { concen- } \\
\text { trations }\end{array}$} & \multirow[b]{2}{*}{ Modelling } & \multirow{2}{*}{$\begin{array}{c}\text { Sam- } \\
\text { pling } \\
\text { methods }\end{array}$} \\
\hline & & & material & $\begin{array}{l}\text { length } \\
{[\mathrm{cm}]}\end{array}$ & $\begin{array}{l}\text { diameter } \\
{[\mathbf{c m}]}\end{array}$ & type & $\begin{array}{l}\text { instal- } \\
\text { lation }\end{array}$ & porosity & & & tubes & pump & fluids & & & \\
\hline $\begin{array}{l}\text { Aga et al. } \\
(2003)\end{array}$ & leaching & tetracycline & \begin{tabular}{|l} 
irrigation \\
pipes \\
with \\
metal \\
screen \\
and po- \\
rous tin \\
cover
\end{tabular} & $\begin{array}{l}\text { ap- } \\
\text { prox. } \\
152\end{array}$ & $\begin{array}{l}\text { approx. } \\
20\end{array}$ & natural soil & $\begin{array}{l}\text { undis- } \\
\text { turbed }\end{array}$ & $\mathrm{n} / \mathrm{a}$ & $n / a$ & $\mathrm{n} / \mathrm{a}$ & $\mathrm{n} / \mathrm{a}$ & $\mathrm{n} / \mathrm{a}$ & distilled water & $\mathrm{n} / \mathrm{a}$ & $\mathrm{n} / \mathrm{a}$ & $\mathrm{n} / \mathrm{a}$ \\
\hline $\begin{array}{l}\text { Cabrera- } \\
\text { Lafaurie et } \\
\text { al. (2015) }\end{array}$ & leaching & $\begin{array}{l}\text { caffeine, } \\
\text { carbamazepine, } \\
\text { clofibric acid, } \\
\text { salicylic acid }\end{array}$ & glass & 30 & 0.8 & $\begin{array}{l}\text { artificial sedi- } \\
\text { ment (inor- } \\
\text { ganic-organic } \\
\text { pillared clays } \\
\text { modified with } \\
\text { transition } \\
\text { metals) }\end{array}$ & $\mathrm{n} / \mathrm{a}$ & $\mathrm{n} / \mathrm{a}$ & $\begin{array}{l}2 \text { and } 7.5 \\
\mathrm{~mL} / \mathrm{min}\end{array}$ & $\mathrm{n} / \mathrm{a}$ & $n / a$ & by gravity & $\begin{array}{l}\text { distilled \& deion- } \\
\text { ized water }\end{array}$ & $14 \mathrm{mg} / \mathrm{L}$ & $\begin{array}{l}\text { fitted to ana- } \\
\text { lytical }\end{array}$ & $\mathrm{n} / \mathrm{a}$ \\
\hline $\begin{array}{l}\text { Cordy et al. } \\
\text { (2004) }\end{array}$ & $\begin{array}{l}\text { leaching } \\
\text { (satu- } \\
\text { rated) }\end{array}$ & $\begin{array}{l}131 \text { organic waste water } \\
\text { compounds and patho- } \\
\text { gens, e.g. antibiotics, } \\
\text { steroids, reproductive } \\
\text { hormones }\end{array}$ & $\begin{array}{l}\text { stainless } \\
\text { steel }\end{array}$ & 240 & 32.5 & natural soil & $\begin{array}{l}\text { hand } \\
\text { packed }\end{array}$ & 0.38 & $\begin{array}{l}16.5 \text { to } \\
4.5 \mathrm{~cm} / \mathrm{d}\end{array}$ & $\begin{array}{l}\text { sand } \\
\text { layer at } \\
\text { the bot- } \\
\text { tom }\end{array}$ & $\mathrm{n} / \mathrm{a}$ & $\mathrm{n} / \mathrm{a}$ & $\begin{array}{l}\text { treated waste } \\
\text { water }\end{array}$ & $\begin{array}{l}26 \mu \mathrm{g} / \mathrm{L} \\
\text { (total } \\
\text { concen- } \\
\text { tration of } \\
\text { all com- } \\
\text { pounds) }\end{array}$ & $\mathrm{n} / \mathrm{a}$ & by hand \\
\hline $\begin{array}{l}\text { De Wilde et } \\
\text { al. (2009) }\end{array}$ & $\begin{array}{l}\text { leaching } \\
\text { (unsatu- } \\
\text { rated) }\end{array}$ & $\begin{array}{l}\text { bentazone, } \\
\text { isoproturon, } \\
\text { linuron, } \\
\text { metalaxyl }\end{array}$ & glass & 15 & 10 & artificial soil & $\begin{array}{l}\text { com- } \\
\text { paction } \\
\text { by a } \\
\text { weight } \\
\text { on top } \\
\text { of the } \\
\text { column }\end{array}$ & $\mathrm{n} / \mathrm{a}$ & $\begin{array}{l}1.74 \mathrm{~cm} / \mathrm{d} \\
\text { (Darcy } \\
\text { flux) }\end{array}$ & glass filter & PTFE & $\begin{array}{l}\text { peristaltic } \\
\text { pump }\end{array}$ & $\begin{array}{l}\text { artificial } \\
\text { (ground)water } \\
\left(\mathrm{CaCl}_{2}\right)\end{array}$ & $\begin{array}{l}10 \mathrm{mg} / \mathrm{L} \\
\text { each } \\
\text { com- } \\
\text { pound }\end{array}$ & $\begin{array}{l}\text { HYDRUS- } \\
1 \mathrm{D}, \mathrm{CDE} \\
\text { (Simunek et } \\
\text { al., 2005) }\end{array}$ & $\begin{array}{l}\text { fraction } \\
\text { collector }\end{array}$ \\
\hline $\begin{array}{l}\text { Dusek et al. } \\
\text { (2015) }\end{array}$ & $\begin{array}{l}\text { Leaching } \\
\text { (satu- } \\
\text { rated) }\end{array}$ & $\begin{array}{l}\text { atrazine, } \\
\text { imazaquin, } \\
\text { sulfometuron methyl, } \\
\text { S-metolachlor, } \\
\text { imidacloprid }\end{array}$ & $n / a$ & 20 & 15 & natural soil & $\begin{array}{l}\text { undis- } \\
\text { turbed }\end{array}$ & $\mathrm{n} / \mathrm{a}$ & $15 \mathrm{~cm} / \mathrm{d}$ & $\begin{array}{l}\text { glass } \\
\text { wool }\end{array}$ & $n / a$ & $\begin{array}{l}\text { high pres- } \\
\text { sure liquid } \\
\text { chroma- } \\
\text { tography } \\
\text { pump }\end{array}$ & $\begin{array}{l}\text { artificial water } \\
\left(\mathrm{CaCl}_{2}\right)\end{array}$ & $\begin{array}{l}5-10 \\
\mathrm{mg} / \mathrm{L}\end{array}$ & $\begin{array}{l}\text { S1D (Vogel } \\
\text { et al., 2007) }\end{array}$ & $\begin{array}{l}\text { fraction } \\
\text { collector }\end{array}$ \\
\hline $\begin{array}{l}\text { Estrella et } \\
\text { al. (1993) }\end{array}$ & leaching & $\begin{array}{l}\text { 2,4-dichlorophenoxyace- } \\
\text { tic acid }(2,4-D)\end{array}$ & glass & 30.5 & 5 & natural soil & $\mathrm{n} / \mathrm{a}$ & $\mathrm{n} / \mathrm{a}$ & $0.7 \mathrm{~cm} / \mathrm{h}$ & $\begin{array}{l}\text { porous } \\
\text { stainless } \\
\text { steel plate } \\
\text { at the bot- } \\
\text { tom of the } \\
\text { glass col- } \\
\text { umn }\end{array}$ & $n / a$ & $\begin{array}{l}\text { vacuum } \\
\text { chamber }\end{array}$ & $\begin{array}{l}\text { artificial } \\
\text { (ground)water } \\
\left(\mathrm{CaCl}_{2}\right)\end{array}$ & $\begin{array}{l}100 \\
\mathrm{mg} / \mathrm{L}\end{array}$ & $\begin{array}{l}\text { numerical } \\
\text { solution of } \\
\text { the CDE, } \\
\text { incl. first or- } \\
\text { der degrada- } \\
\text { tion (Van } \\
\text { Genuchten } \\
\text { and } \\
\text { Wagenet, } \\
\text { 1989) }\end{array}$ & $\begin{array}{l}\text { fraction } \\
\text { collector }\end{array}$ \\
\hline
\end{tabular}




\begin{tabular}{|c|c|c|c|c|c|c|c|c|c|c|c|c|c|c|c|c|}
\hline \multirow[b]{2}{*}{ Citation } & \multirow{2}{*}{$\begin{array}{l}\text { Condi- } \\
\text { tions }\end{array}$} & \multirow[b]{2}{*}{ Compounds } & \multicolumn{3}{|c|}{ Column } & \multicolumn{3}{|c|}{ Sediment } & \multirow{2}{*}{$\begin{array}{c}\text { Veloci- } \\
\text { ties / } \\
\text { flowrates }\end{array}$} & \multirow{2}{*}{$\begin{array}{l}\text { Filter lay- } \\
\text { ers }\end{array}$} & \multicolumn{3}{|c|}{ Infrastructure / used materials } & \multirow{2}{*}{\begin{tabular}{|l|} 
Input \\
concen- \\
trations
\end{tabular}} & \multirow[b]{2}{*}{ Modelling } & \multirow{2}{*}{$\begin{array}{c}\text { Sam- } \\
\text { pling } \\
\text { methods }\end{array}$} \\
\hline & & & material & $\begin{array}{c}\text { length } \\
{[\mathrm{cm}]}\end{array}$ & $\begin{array}{l}\text { diameter } \\
{[\mathrm{cm}]}\end{array}$ & type & $\begin{array}{l}\text { instal- } \\
\text { lation }\end{array}$ & porosity & & & tubes & pump & fluids & & & \\
\hline $\begin{array}{l}\text { Fan et al. } \\
\text { (2011) }\end{array}$ & leaching & sulfamethazine & glass & 15 & 8.4 & $\begin{array}{l}\text { natural soil } \\
\text { (garden soil), } \\
\text { artificial sedi- } \\
\text { ment (filter } \\
\text { sand / tech- } \\
\text { nical quartz } \\
\text { sand) }\end{array}$ & $\begin{array}{l}\text { dry, } \\
\text { wetted } \\
\text { from the } \\
\text { bottom }\end{array}$ & $\begin{array}{l}0.37-0.67 \\
\text { (volumet- } \\
\text { ric water } \\
\text { content) }\end{array}$ & $\begin{array}{l}19.8- \\
39.5 \mathrm{~cm} / \mathrm{h}\end{array}$ & $\begin{array}{l}\text { stainless } \\
\text { steel } \\
\text { mesh, } \\
\text { cheese- } \\
\text { cloth }\end{array}$ & $\begin{array}{l}\text { Teflon } \\
\text { (PTFE) }\end{array}$ & $\mathrm{n} / \mathrm{a}$ & $\begin{array}{l}\text { artificial } \\
\text { (ground)water } \\
\left(\mathrm{CaCl}_{2}\right)\end{array}$ & $8 \mu \mathrm{g} / \mathrm{L}$ & $\begin{array}{l}\text { CXTFIT, } \\
\text { CDE (Toride } \\
\text { et al., 1995), } \\
\text { libSRES (Ji } \\
\text { and Xu, } \\
\text { 2006) }\end{array}$ & $\begin{array}{l}\text { fraction } \\
\text { collector }\end{array}$ \\
\hline $\begin{array}{l}\text { Kamra et al. } \\
(2001)\end{array}$ & $\begin{array}{l}\text { leaching } \\
\text { (unsatu- } \\
\text { rated) }\end{array}$ & $\begin{array}{l}\text { atrazine, } \\
\text { isoproturon }\end{array}$ & $n / a$ & 10 & 5.7 & natural soil & $\begin{array}{l}\text { undis- } \\
\text { turbed }\end{array}$ & $\mathrm{n} / \mathrm{a}$ & $\begin{array}{l}0.8 \mathrm{~cm} / \mathrm{d} \\
(\text { Darcy } \\
\text { flux) } \\
1.92- \\
2.48 \mathrm{~cm} / \mathrm{d} \\
\text { (pore wa- } \\
\text { ter veloc- } \\
\text { ity) }\end{array}$ & $\begin{array}{l}\text { porous } \\
\text { glass } \\
\text { plate with } \\
\text { nylon } \\
\text { mem- } \\
\text { brane }\end{array}$ & $n / a$ & $\begin{array}{l}\text { vacuum } \\
\text { pump }\end{array}$ & artificial rain & $n / a$ & $\begin{array}{l}\text { CXTFIT, } \\
\text { CDE (Toride } \\
\text { et al., 1995), } \\
\text { time moment } \\
\text { analysis } \\
\text { (Jury and } \\
\text { Sposito, } \\
\text { 1985) }\end{array}$ & $\begin{array}{l}\text { fraction } \\
\text { collector }\end{array}$ \\
\hline $\begin{array}{l}\text { Kay et al. } \\
(2005)\end{array}$ & $\begin{array}{l}\text { leaching } \\
\text { (unsatu- } \\
\text { rated) }\end{array}$ & $\begin{array}{l}\text { oxytetracycline, } \\
\text { sulphachloropyridazine, } \\
\text { tylosin }\end{array}$ & $n / a$ & 30 & 5 & $\begin{array}{l}\text { natural soil, } \\
\text { partly pig } \\
\text { farm slurry on } \\
\text { top }\end{array}$ & $\begin{array}{l}\text { undis- } \\
\text { turbed } \\
\& \text { dis- } \\
\text { turbed } \\
\text { (air } \\
\text { dried \& } \\
\text { sieved) }\end{array}$ & $\mathrm{n} / \mathrm{a}$ & $\begin{array}{l}45000 \\
\mathrm{~L} / \mathrm{ha}\end{array}$ & $\begin{array}{l}\text { nylon } \\
\text { mesh }\end{array}$ & $\begin{array}{l}\text { HDPE } \\
\text { funnel }\end{array}$ & $\mathrm{n} / \mathrm{a}$ & $\begin{array}{l}\text { artificial } \\
\text { (ground)water } \\
\left(\mathrm{CaCl}_{2}\right)\end{array}$ & $\begin{array}{l}18.85- \\
25.58 \mathrm{~m} \\
\mathrm{~g} / \mathrm{L}\end{array}$ & $\mathrm{n} / \mathrm{a}$ & $\begin{array}{l}\text { amber } \\
\text { glass bot- } \\
\text { tles }\end{array}$ \\
\hline $\begin{array}{l}\text { Lopez- } \\
\text { Blanco et } \\
\text { al. (2005) }\end{array}$ & $\begin{array}{l}\text { leaching } \\
\text { (unsatu- } \\
\text { rated) }\end{array}$ & a-endosulfan & PVC & 80 & 1.5 & $\begin{array}{l}\text { natural sedi- } \\
\text { ment, natural } \\
\text { soil }\end{array}$ & $\mathrm{n} / \mathrm{a}$ & 0.49 & $1.14 \mathrm{~cm} / \mathrm{h}$ & $\begin{array}{l}\text { washed } \\
\text { quartz } \\
\text { sand, } \\
0.25-0.5 \\
\mathrm{~mm}\end{array}$ & $\begin{array}{l}\text { PTFE, } \\
\text { fluoro- } \\
\text { elasto- } \\
\text { mer }\end{array}$ & $\begin{array}{l}\text { peristaltic } \\
\text { pump }\end{array}$ & $\begin{array}{l}\text { artificial } \\
\text { (ground)water } \\
(\mathrm{KCl})\end{array}$ & $\begin{array}{l}100 \\
\mathrm{mg} / \mathrm{L}\end{array}$ & $\begin{array}{l}\text { CXTFIT, } \\
\text { CDE (Toride } \\
\text { et al., 1999), } \\
\text { CHAIN (Van } \\
\text { Genuchten, } \\
\text { 1985) }\end{array}$ & $\mathrm{n} / \mathrm{a}$ \\
\hline $\begin{array}{l}\text { Maeng et } \\
\text { al. (2011) }\end{array}$ & $\begin{array}{l}\text { n/a (prob- } \\
\text { ably } \\
\text { leaching) }\end{array}$ & $\begin{array}{l}\text { bezafibrate, } \\
\text { caffeine, } \\
\text { carbamazepine, } \\
\text { clofibric acid, } \\
\text { gemfibrozil, } \\
\text { diclofenac, } \\
\text { fenoprofen, } \\
\text { ibuprofen, } \\
\text { ketoprofen, } \\
\text { naproxen, } \\
\text { paracetamol, } \\
\text { pentoxifylline, } \\
\text { phenacetine }\end{array}$ & $\begin{array}{l}\text { n/a } \\
\text { ("XK50/3 } \\
0 ; \text { Amer- } \\
\text { sham } \\
\text { Pharma- } \\
\text { cia Bio- } \\
\text { tech, } \\
\text { Swe- } \\
\text { den") }\end{array}$ & 30 & 5 & $\begin{array}{l}\text { artificial sedi- } \\
\text { ment (filter } \\
\text { sand / tech- } \\
\text { nical quartz } \\
\text { sand) }\end{array}$ & $\mathrm{n} / \mathrm{a}$ & $\mathrm{n} / \mathrm{a}$ & $\begin{array}{l}0.64 \mathrm{~m} / \mathrm{d} \\
\text { (hydraulic } \\
\text { loading } \\
\text { rate) }\end{array}$ & $\mathrm{n} / \mathrm{a}$ & $n / a$ & $\mathrm{n} / \mathrm{a}$ & $\begin{array}{l}\text { surface water, } \\
\text { tap water, } \\
\text { treated waste } \\
\text { water, deminer- } \\
\text { alized water }\end{array}$ & $\begin{array}{l}1.2-8.1 \\
\mu \mathrm{g} / \mathrm{L}\end{array}$ & $\mathrm{n} / \mathrm{a}$ & $\mathrm{n} / \mathrm{a}$ \\
\hline $\begin{array}{l}\text { Murillo- } \\
\text { Torres et al. } \\
\text { (2012) }\end{array}$ & $\begin{array}{l}\text { leaching } \\
\text { (unsatu- } \\
\text { rated) }\end{array}$ & $\begin{array}{l}\text { 4-nonylphenol, } \\
\text { di-2- } \\
\text { ethyl(hexyl)phthalate }\end{array}$ & $\mathrm{n} / \mathrm{a}$ & 15 & 3 & natural soil & moist & na & $\begin{array}{l}0.013 \\
\mathrm{~mL} / \mathrm{s}\end{array}$ & $\mathrm{n} / \mathrm{a}$ & $n / a$ & $\begin{array}{l}\text { peristaltic } \\
\text { pump }\end{array}$ & $\begin{array}{l}\text { artificial } \\
\text { (ground)water } \\
\left(\mathrm{CaCl}_{2}\right)\end{array}$ & $n / a$ & $\begin{array}{l}\text { temporal mo- } \\
\text { ments (Pang } \\
\text { et al., 2003) }\end{array}$ & $\begin{array}{l}\text { by gravity } \\
\text { in pre- } \\
\text { cleaned } \\
\text { glass } \\
\text { flask }\end{array}$ \\
\hline
\end{tabular}




\begin{tabular}{|c|c|c|c|c|c|c|c|c|c|c|c|c|c|c|c|c|}
\hline \multirow{2}{*}{ Citation } & \multirow{2}{*}{$\begin{array}{l}\text { Condi- } \\
\text { tions }\end{array}$} & \multirow{2}{*}{ Compounds } & \multicolumn{3}{|c|}{ Column } & \multicolumn{3}{|c|}{ Sediment } & \multirow{2}{*}{\begin{tabular}{|c|} 
Veloci- \\
ties / \\
flowrates
\end{tabular}} & \multirow{2}{*}{$\begin{array}{l}\text { Filter lay- } \\
\text { ers }\end{array}$} & \multicolumn{3}{|c|}{ Infrastructure / used materials } & \multirow{2}{*}{$\begin{array}{l}\text { Input } \\
\text { concen- } \\
\text { trations }\end{array}$} & \multirow{2}{*}{ Modelling } & \multirow{2}{*}{$\begin{array}{c}\text { Sam- } \\
\text { pling } \\
\text { methods }\end{array}$} \\
\hline & & & material & $\begin{array}{c}\text { length } \\
{[\mathrm{cm}]}\end{array}$ & $\begin{array}{c}\text { diameter } \\
{[\mathrm{cm}]}\end{array}$ & type & $\begin{array}{l}\text { instal- } \\
\text { lation }\end{array}$ & porosity & & & tubes & pump & fluids & & & \\
\hline $\begin{array}{l}\text { Nkedi-Kizza } \\
\text { et al. (1987) }\end{array}$ & $\begin{array}{l}\text { leaching } \\
\text { (satu- } \\
\text { rated) }\end{array}$ & $\begin{array}{l}\text { atrazine, } \\
\text { diuron }\end{array}$ & $\begin{array}{l}\text { glass } \\
\text { (HPLC- } \\
\text { column) }\end{array}$ & 30 & 2.5 & $\begin{array}{l}\text { natural soil } \\
\text { (Eustis soil) }\end{array}$ & air-dry & $\begin{array}{l}0.33-0.41 \\
\text { (as water } \\
\text { content) }\end{array}$ & $\begin{array}{l}5.18- \\
6.44 \mathrm{~cm} / \mathrm{h}\end{array}$ & $\mathrm{n} / \mathrm{a}$ & $\begin{array}{l}\text { HPLC } \\
\text { fittings }\end{array}$ & $\begin{array}{l}\text { HPLC } \\
\text { pump }\end{array}$ & $\begin{array}{l}\text { aqueous solu- } \\
\text { tions from } 0.01 \\
\mathrm{~N} \mathrm{CaCl} 2 \text { \&ari- } \\
\text { ous methanol- } \\
\text { water mixtures }\end{array}$ & $\begin{array}{l}30 \mathrm{mg} / \mathrm{L} \\
\text { diuron } \\
20 \mathrm{mg} / \mathrm{L} \\
\text { atrazine }\end{array}$ & $\begin{array}{l}\text { leaching re- } \\
\text { tardation fac- } \\
\text { tors by } \\
\text { graphical } \\
\text { analysis of } \\
\text { column } \\
\text { BTCs }\end{array}$ & $\begin{array}{l}\text { flow- } \\
\text { through } \\
\text { variable- } \\
\text { wave- } \\
\text { length UV } \\
\text { detector, } \\
\text { assaying } \\
\text { radioac- } \\
\text { tivity with } \\
\text { liquid } \\
\text { scintilla- } \\
\text { tion tech- } \\
\text { niques }\end{array}$ \\
\hline $\begin{array}{l}\text { Oppel et al. } \\
\text { (2004) }\end{array}$ & $\begin{array}{l}\text { Leaching } \\
\text { according } \\
\text { to OECD } \\
\text { (2003) } \\
\text { guideline } \\
\text { (unsatu- } \\
\text { rated) }\end{array}$ & $\begin{array}{l}\text { carbamazepine, } \\
\text { clofibric acid, } \\
\text { diazepam, } \\
\text { ibuprofen, } \\
\text { ivermectin, } \\
\text { iopromide and its deriva- } \\
\text { tives, } \\
\text { 5-amino-2,4,6-triiodo- } \\
\text { isophthalic acid, } \\
\text { desmethoxyacetyl- } \\
\text { iopromide, } \\
\text { (N-2,3-dihydroxypropyl)- } \\
\text { 5-amino-2,4,6-triiodo- } \\
\text { isophthalic acid amide }\end{array}$ & glass & 30 & $\mathrm{n} / \mathrm{a}$ & natural soil & $\begin{array}{l}\text { air-dry, } \\
\text { com-- } \\
\text { paction } \\
\text { by vi- } \\
\text { bration }\end{array}$ & $\mathrm{n} / \mathrm{a}$ & $\begin{array}{l}393 \mathrm{~mL} \\
\text { drop wise } \\
\text { within } 48 \\
\mathrm{~h}\end{array}$ & $\mathrm{n} / \mathrm{a}$ & $n / a$ & $\mathrm{n} / \mathrm{a}$ & $\begin{array}{l}\text { artificial rain } \\
(0.01 \mathrm{~mol} / \mathrm{L} \\
\left(\mathrm{CaCl}_{2}\right)\end{array}$ & $\begin{array}{l}\text { as aque- } \\
\text { ous solu- } \\
\text { tions or } \\
\text { dis- } \\
\text { solved in } \\
\text { organic } \\
\text { solvent: } \\
100 \\
\mathrm{mg} / \mathrm{kg} \\
\text { soil (dry } \\
\text { weight) }\end{array}$ & $\mathrm{n} / \mathrm{a}$ & $\mathrm{n} / \mathrm{a}$ \\
\hline $\begin{array}{l}\text { Rodriguez- } \\
\text { Cruz et al. } \\
\text { (2007) }\end{array}$ & $\begin{array}{l}\text { leaching } \\
\text { (saturated } \\
\text { flow re- } \\
\text { gime) }\end{array}$ & $\begin{array}{l}\text { atrazine, } \\
\text { linuron, } \\
\text { metalaxyl }\end{array}$ & glass & 20 & 3 & $\begin{array}{l}\text { natural soil, } \\
\text { partly ex \& in } \\
\text { situ modified } \\
\text { with cationic } \\
\text { surfactant oc- } \\
\text { tadecyltrime- } \\
\text { thylammoni- } \\
\text { umbromde }\end{array}$ & dry & $\mathrm{n} / \mathrm{a}$ & $\begin{array}{l}\text { drainage } \\
\text { rate of } 1 \\
\mathrm{~mL} / \mathrm{min}\end{array}$ & $\mathrm{n} / \mathrm{a}$ & $n / a$ & $\begin{array}{l}\text { peristaltic } \\
\text { pump }\end{array}$ & $\mathrm{n} / \mathrm{a}$ & $\begin{array}{l}1000 \\
\mu \mathrm{g} / \mathrm{L} \text { in } \\
\text { metha- } \\
\text { nol }(1 \\
\mathrm{mL} \text { of } \\
\text { solution } \\
\text { applied } \\
\text { at the } \\
\text { top of } \\
\text { the soil } \\
\text { and } \\
\text { leached } \\
\text { by appli- } \\
\text { cation of } \\
500 \mathrm{~mL} \\
\text { of water) }\end{array}$ & $\mathrm{n} / \mathrm{a}$ & $\begin{array}{l}\text { fraction } \\
\text { collector }\end{array}$ \\
\hline
\end{tabular}




\begin{tabular}{|c|c|c|c|c|c|c|c|c|c|c|c|c|c|c|c|c|}
\hline \multirow{2}{*}{ Citation } & \multirow{2}{*}{$\begin{array}{l}\text { Condi- } \\
\text { tions }\end{array}$} & \multirow{2}{*}{ Compounds } & \multicolumn{3}{|c|}{ Column } & \multicolumn{3}{|c|}{ Sediment } & \multirow{2}{*}{$\begin{array}{l}\text { Veloci- } \\
\text { ties / } \\
\text { flowrates }\end{array}$} & \multirow{2}{*}{$\begin{array}{l}\text { Filter lay- } \\
\text { ers }\end{array}$} & \multicolumn{3}{|c|}{ Infrastructure / used materials } & \multirow{2}{*}{$\begin{array}{l}\text { Input } \\
\text { concen- } \\
\text { trations }\end{array}$} & \multirow{2}{*}{ Modelling } & \multirow{2}{*}{$\begin{array}{c}\text { Sam- } \\
\text { pling } \\
\text { methods }\end{array}$} \\
\hline & & & material & $\begin{array}{c}\text { length } \\
{[\mathrm{cm}]}\end{array}$ & $\begin{array}{l}\text { diameter } \\
{[\mathrm{cm}]}\end{array}$ & type & $\begin{array}{l}\text { instal- } \\
\text { lation }\end{array}$ & porosity & & & tubes & pump & fluids & & & \\
\hline $\begin{array}{l}\text { Salem Attia } \\
\text { et al. (2013) }\end{array}$ & $\begin{array}{l}\text { leaching } \\
\text { (satu- } \\
\text { rated) }\end{array}$ & $\begin{array}{l}\text { diclofenac, } \\
\text { gemfibrozil, } \\
\text { ibuprofen, } \\
\text { naproxen }\end{array}$ & glass & 25 & 1 & $\begin{array}{l}\text { artificial sedi- } \\
\text { ment (mag- } \\
\text { netic nano- } \\
\text { particle } \\
\text { coated zeo- } \\
\text { lithe) }\end{array}$ & $\mathrm{n} / \mathrm{a}$ & $\mathrm{n} / \mathrm{a}$ & \begin{tabular}{|l|} 
flow rate: \\
25 \\
$\mathrm{~mL} / \mathrm{min}$
\end{tabular} & $\begin{array}{l}\text { glass } \\
\text { wool }\end{array}$ & $n / a$ & $\begin{array}{l}\text { suction } \\
\text { pump }\end{array}$ & $\begin{array}{l}\text { influent from } \\
\text { drinking water } \\
\text { treatment plants }\end{array}$ & $n / a$ & $\mathrm{n} / \mathrm{a}$ & $\begin{array}{l}1 \mathrm{~L} \text { am- } \\
\text { ber glass } \\
\text { bottles. }\end{array}$ \\
\hline $\begin{array}{l}\text { Salvia et al. } \\
\text { (2014) }\end{array}$ & $\begin{array}{l}\text { leaching } \\
\text { (unsatu- } \\
\text { rated) }\end{array}$ & $\begin{array}{l}\text { androstenedione, } \\
\text { carbamazepine, } \\
\text { dicyclanil, } \\
\text { erythromycin, } \\
\text { fluvoxamine, } \\
\text { gestodene, } \\
\text { levonorgestrel, } \\
\text { norethindrone, } \\
\text { paracetamol, } \\
\text { penicillin G potassium } \\
\text { salt, } \\
\text { progesterone, } \\
\text { roxithromycin, } \\
\text { sulphabenzamide, } \\
\text { sulphadiazine, } \\
\text { sulphadimethoxine, } \\
\text { sulphadimidine, } \\
\text { sulphameter, } \\
\text { sulphamethoxazole, } \\
\text { sulphanilamide, } \\
\text { sulphathiazole, } \\
\text { testosterone, } \\
\text { trimethoprim, } \\
\text { tylosin tartrate }\end{array}$ & PVC & 30 & 10 & natural soil & dry & $n / a$ & $\begin{array}{l}40 \mathrm{~mL} \text { of } \\
\text { artificial } \\
\text { rain every } \\
2 \text { days }\end{array}$ & $\begin{array}{l}\text { glass } \\
\text { beads }\end{array}$ & $n / a$ & $n / a$ & $\begin{array}{l}\text { artificial rain ( } \\
\left.\mathrm{CaCl}_{2}\right)\end{array}$ & $\begin{array}{l}1 \mathrm{mg} \text { of } \\
\text { each } \\
\text { sub- } \\
\text { stance }\end{array}$ & $\begin{array}{l}\text { fitting of deg- } \\
\text { radation } \\
\text { curves to the } \\
\text { exponential } \\
\text { decay model }\end{array}$ & $\begin{array}{l}\text { leachates } \\
\text { were re- } \\
\text { covered } \\
\text { every } 2 \\
\text { days in } \\
\text { amber } \\
\text { flasks }\end{array}$ \\
\hline $\begin{array}{l}\text { Schaffer et } \\
\text { al. (2015) }\end{array}$ & $\begin{array}{l}\text { leaching } \\
\text { (varying } \\
\text { hydraulic } \\
\text { recharge } \\
\text { condi- } \\
\text { tions) }\end{array}$ & $\begin{array}{l}\text { 4-acetaminoantipyrine, } \\
\text { 1H-benzotriazole, } \\
\text { acesulfame, } \\
\text { atenolol, } \\
\text { atenolol acid, } \\
\text { bezafibrate, } \\
\text { carbamazepine, } \\
\text { citalopram, } \\
\text { clarithromycin, } \\
\text { diclofenac, } \\
\text { diazepam, } \\
\text { fluoxetine, } \\
\text { gemfibrozil, } \\
\text { haloperidol, } \\
\text { ibuprofen, } \\
\text { irbesartan, } \\
\text { isoproturon, } \\
\text { losartan, mecoprop, } \\
\text { metoprolol, } \\
\text { naproxen, }\end{array}$ & $\begin{array}{l}\text { stainless } \\
\text { steel }\end{array}$ & 150 & 35 & $\begin{array}{l}\text { artificial sedi- } \\
\text { ment (mix } \\
\text { from sieved } \\
\text { sand and } \\
\text { compost) }\end{array}$ & $\mathrm{n} / \mathrm{a}$ & $0.40-0.49$ & $\begin{array}{l}0.05- \\
0.19 \mathrm{~m} / \mathrm{d}\end{array}$ & $\mathrm{n} / \mathrm{a}$ & $\begin{array}{l}\text { PTFE, } \\
\text { stain- } \\
\text { less } \\
\text { steel }\end{array}$ & $\begin{array}{l}\text { peristaltic } \\
\text { pump }\end{array}$ & $\begin{array}{l}\text { treated waste } \\
\text { water }\end{array}$ & $\begin{array}{l}\text { up to } 6.5 \\
\mu \mathrm{g} / \mathrm{L}\end{array}$ & $\begin{array}{l}\text { CXTFIT, } \\
\text { CDE (Toride } \\
\text { et al., 1995) } \\
\text { for tracer } \\
\text { BTCs, } \\
\text { PMWIN with } \\
\text { MT3D } \\
\text { (Chiang and } \\
\text { Kinzelbach, } \\
\text { 1998) for } \\
\text { compound } \\
\text { BTCs }\end{array}$ & $\begin{array}{l}\text { glass sy- } \\
\text { ringe }\end{array}$ \\
\hline
\end{tabular}




\begin{tabular}{|c|c|c|c|c|c|c|c|c|c|c|c|c|c|c|c|c|}
\hline \multirow[b]{2}{*}{ Citation } & \multirow{2}{*}{$\begin{array}{l}\text { Condi- } \\
\text { tions }\end{array}$} & \multirow[b]{2}{*}{ Compounds } & \multicolumn{3}{|c|}{ Column } & \multicolumn{3}{|c|}{ Sediment } & \multirow{2}{*}{$\begin{array}{c}\text { Veloci- } \\
\text { ties / } \\
\text { flowrates }\end{array}$} & \multirow{2}{*}{$\begin{array}{l}\text { Filter lay- } \\
\text { ers }\end{array}$} & \multicolumn{3}{|c|}{ Infrastructure / used materials } & \multirow{2}{*}{\begin{tabular}{|c|} 
Input \\
concen- \\
trations
\end{tabular}} & \multirow[b]{2}{*}{ Modelling } & \multirow{2}{*}{$\begin{array}{c}\text { Sam- } \\
\text { pling } \\
\text { methods }\end{array}$} \\
\hline & & & material & $\begin{array}{c}\text { length } \\
{[\mathrm{cm}]}\end{array}$ & $\begin{array}{l}\text { diameter } \\
{[\mathrm{cm}]}\end{array}$ & type & $\begin{array}{l}\text { instal- } \\
\text { lation }\end{array}$ & porosity & & & tubes & pump & fluids & & & \\
\hline & & $\begin{array}{l}\text { phenazone, primidone, } \\
\text { sulfamethoxazole, } \\
\text { tamoxifen, } \\
\text { tolyltriazole, } \\
\text { valsartan, } \\
\text { valsartan acid }\end{array}$ & & & & & & & & & & & & & & \\
\hline $\begin{array}{l}\text { Scheytt et } \\
\text { al. (2006) }\end{array}$ & $\begin{array}{l}\text { leaching } \\
\text { (unsatu- } \\
\text { rated) }\end{array}$ & $\begin{array}{l}\text { carbamazepine, } \\
\text { diclofenac, } \\
\text { ibuprofen, } \\
\text { propyphenazone }\end{array}$ & $\begin{array}{l}\text { stainless } \\
\text { steel }\end{array}$ & 35 & 13.6 & $\begin{array}{l}\text { natural sedi- } \\
\text { ment }\end{array}$ & $\begin{array}{l}\text { dry, } \\
\text { com- } \\
\text { paction } \\
\text { by } \\
\text { plunger }\end{array}$ & $\mathrm{n} / \mathrm{a}$ & $\begin{array}{l}0.71- \\
0.88 \mathrm{~m} / \mathrm{d} \\
\text { (av pore } \\
\text { water ve- } \\
\text { locities) }\end{array}$ & $\begin{array}{l}\text { glass } \\
\text { beads } \\
\text { and teflon } \\
\text { gauze net } \\
\text { (personal } \\
\text { communi- } \\
\text { cation) }\end{array}$ & $n / a$ & by gravity & $\begin{array}{l}\text { artificial } \\
\text { (ground)water } \\
\text { and simulated } \\
\text { treated waste } \\
\text { water }\end{array}$ & $1 \mu \mathrm{g} / \mathrm{L}$ & $\begin{array}{l}\text { CXTFIT, } \\
\text { CDE (Toride } \\
\text { et al., 1995) }\end{array}$ & $\begin{array}{l}\text { flowthrou } \\
\text { gh cells, } \\
\text { fraction } \\
\text { collector }\end{array}$ \\
\hline $\begin{array}{l}\text { Scheytt et } \\
\text { al. (2007) }\end{array}$ & $\begin{array}{l}\text { leaching } \\
\text { (unsatu- } \\
\text { rated) }\end{array}$ & $\begin{array}{l}\text { clofibric acid, } \\
\text { diclofenac, } \\
\text { ibuprofen, } \\
\text { propyphenazone }\end{array}$ & $\begin{array}{l}\text { stainless } \\
\text { steel }\end{array}$ & 35 & 13.6 & $\begin{array}{l}\text { natural sedi- } \\
\text { ment }\end{array}$ & $\begin{array}{l}\text { dry, } \\
\text { com- } \\
\text { paction } \\
\text { by } \\
\text { plunger }\end{array}$ & $n / a$ & $\begin{array}{l}0.96 \mathrm{~m} / \mathrm{d} \\
\text { (av pore } \\
\text { water ve- } \\
\text { locities) }\end{array}$ & $\begin{array}{l}\text { glass } \\
\text { beads } \\
\text { and teflon } \\
\text { gauze net } \\
\text { (personal } \\
\text { communi- } \\
\text { cation) }\end{array}$ & $n / a$ & by gravity & $\begin{array}{l}\text { artificial } \\
\text { (ground)water - } \\
\text { simulated } \\
\text { treated waste } \\
\text { water }\end{array}$ & $1 \mu \mathrm{g} / \mathrm{L}$ & $\begin{array}{l}\text { CXTFIT, } \\
\text { CDE (Toride } \\
\text { et al., 1995) }\end{array}$ & $\begin{array}{l}\text { flowthrou } \\
\text { gh cells, } \\
\text { fraction } \\
\text { collector }\end{array}$ \\
\hline $\begin{array}{l}\text { Siemens et } \\
\text { al. (2010) }\end{array}$ & leaching & $\begin{array}{l}\text { bezafibrate, } \\
\text { clarithromycin, } \\
\text { clindamycin, } \\
\text { diclofenac, } \\
\text { erythromycin, } \\
\text { gemfibrozil, } \\
\text { ibuprofen, } \\
\text { metoprolol, } \\
\text { naproxen, } \\
\text { trimethoprim }\end{array}$ & PVC & 10 & 8 & natural soil & $\mathrm{n} / \mathrm{a}$ & $\mathrm{n} / \mathrm{a}$ & $\begin{array}{l}0.047 \\
\mathrm{~cm} / \mathrm{h}- \\
0.136 \\
\mathrm{~cm} / \mathrm{h}\end{array}$ & $\begin{array}{l}\text { moist } \\
\text { quartz silt, } \\
\text { porous } \\
\text { glass suc- } \\
\text { tion plate }\end{array}$ & $n / a$ & $\begin{array}{l}\text { suction / } \\
\text { vacuum } \\
\text { pump }\end{array}$ & $\begin{array}{l}\text { artificial } \\
\text { (ground)water, } \\
\text { equilibrated with } \\
\mathrm{CaCO}_{3} \text {, un- } \\
\text { known composi- } \\
\text { tion }\end{array}$ & $\begin{array}{l}20- \\
2000 \\
\mu \mathrm{g} / \mathrm{L}\end{array}$ & $\begin{array}{l}\text { CXTFIT, } \\
\text { CDE (Toride } \\
\text { et al., 1995), } \\
\text { HYDRUS } \\
\text { (Simunek et } \\
\text { al., 1998), } \\
\text { Gaussian 03 } \\
\text { (Frisch et al., } \\
\text { 2004) }\end{array}$ & $\begin{array}{l}\text { aliquots } \\
\text { of } 0.07 \mathrm{~L}\end{array}$ \\
\hline $\begin{array}{l}\text { Unold et al. } \\
\text { (2009) }\end{array}$ & $\begin{array}{l}\text { leaching, } \\
\text { steady } \\
\text { state flow, } \\
\text { near satu- } \\
\text { ration }\end{array}$ & sulfadiazin & $\begin{array}{l}\text { stainless } \\
\text { steel }\end{array}$ & 10 & 8.5 & natural soil & $\begin{array}{l}\text { dry } \\
\text { (slightly } \\
\text { wetted } \\
\text { before } \\
\text { pack- } \\
\text { ing), } \\
\text { com- } \\
\text { paction } \\
\text { by pes- } \\
\text { tle }\end{array}$ & $n / a$ & $\begin{array}{l}0.24- \\
0.26 \mathrm{~cm} / \mathrm{h} \\
\text { (Darcy } \\
\text { velocity) } \\
0.49- \\
0.64 \mathrm{~cm} / \mathrm{h} \\
\text { (tracer } \\
\text { pore ve- } \\
\text { locity) }\end{array}$ & $\begin{array}{l}\text { quartz fil- } \\
\text { ter sand / } \\
\text { gravel, } \\
\text { porous } \\
\text { ceramic } \\
\text { plate }\end{array}$ & $n / a$ & $\begin{array}{l}\text { peristaltic } \\
\text { pump }\end{array}$ & $\begin{array}{l}\text { artificial } \\
\text { (ground)water } \\
\left(\mathrm{CaCl}_{2}\right)\end{array}$ & $\begin{array}{l}0.57 \\
\mathrm{mg} / \mathrm{L}\end{array}$ & $\begin{array}{l}\text { CXTFIT, } \\
\text { CDE (Toride } \\
\text { et al., 1999) } \\
\text { HYDRUS } \\
\text { (Simunek et } \\
\text { al., 1998), } \\
\text { PEST } \\
\text { (Doherty, } \\
\text { 2002) }\end{array}$ & $\begin{array}{l}\text { fraction } \\
\text { collector }\end{array}$ \\
\hline
\end{tabular}




\begin{tabular}{|c|c|c|c|c|c|c|c|c|c|c|c|c|c|c|c|c|}
\hline \multirow[b]{2}{*}{ Citation } & \multirow{2}{*}{$\begin{array}{l}\text { Condi- } \\
\text { tions }\end{array}$} & \multirow[b]{2}{*}{ Compounds } & \multicolumn{3}{|c|}{ Column } & \multicolumn{3}{|c|}{ Sediment } & \multirow{2}{*}{$\begin{array}{c}\text { Veloci- } \\
\text { ties / } \\
\text { flowrates }\end{array}$} & \multirow{2}{*}{$\begin{array}{c}\text { Filter lay- } \\
\text { ers }\end{array}$} & \multicolumn{3}{|c|}{ Infrastructure / used materials } & \multirow{2}{*}{$\begin{array}{c}\text { Input } \\
\text { concen- } \\
\text { trations }\end{array}$} & \multirow[b]{2}{*}{ Modelling } & \multirow{2}{*}{$\begin{array}{c}\text { Sam- } \\
\text { pling } \\
\text { methods }\end{array}$} \\
\hline & & & material & $\begin{array}{c}\text { length } \\
{[\mathrm{cm}]}\end{array}$ & $\begin{array}{c}\text { diameter } \\
{[\mathrm{cm}]}\end{array}$ & type & $\begin{array}{l}\text { instal- } \\
\text { lation }\end{array}$ & porosity & & & tubes & pump & fluids & & & \\
\hline $\begin{array}{l}\text { Wu et al. } \\
(2010)\end{array}$ & $\begin{array}{l}\text { leaching } \\
\text { (unsatu- } \\
\text { rated) }\end{array}$ & $\begin{array}{l}\text { carbamazepine, } \\
\text { carbamazepine-10,11- } \\
\text { epoxide, } \\
\text { clindamycin, } \\
\text { diltiazem, } \\
\text { diphenhydramine, } \\
\text { fluoxetine, } \\
\text { norfluoxetine }\end{array}$ & PVC & 40 & 4 & $\begin{array}{l}\text { natural soil, } \\
\text { biosolids } \\
\text { added }\end{array}$ & $\begin{array}{l}\text { com- } \\
\text { paction } \\
\text { by vi- } \\
\text { bration }\end{array}$ & $\mathrm{n} / \mathrm{a}$ & $\begin{array}{l}200 \mathrm{~mm} \\
\text { over } 48 \mathrm{~h}\end{array}$ & $\begin{array}{l}\text { glass } \\
\text { wool, } \\
\text { quartz fil- } \\
\text { ter sand } \\
\text { and (un- } \\
\text { known) } \\
\text { screening } \\
\text { material }\end{array}$ & $n / a$ & $\begin{array}{l}\text { peristaltic } \\
\text { pump }\end{array}$ & $\begin{array}{l}\text { artificial rain } \\
\left(\mathrm{CaCl}_{2}\right)\end{array}$ & $\begin{array}{l}100 \mathrm{ng} / \mathrm{g} \\
\text { (sub- } \\
\text { stance / } \\
\text { soil) }\end{array}$ & $\mathrm{n} / \mathrm{a}$ & $\begin{array}{l}\text { by glass } \\
\text { funnels } \\
\text { into 250- } \\
\text { ml Erlen- } \\
\text { meyer } \\
\text { flasks } \\
\text { wrapped } \\
\text { with alu- } \\
\text { minum } \\
\text { foil to } \\
\text { avoid } \\
\text { photo- } \\
\text { degrada- } \\
\text { tion }\end{array}$ \\
\hline $\begin{array}{l}\text { Xu et al. } \\
\text { (2010) }\end{array}$ & $\begin{array}{l}\text { leaching } \\
\text { (satu- } \\
\text { rated) }\end{array}$ & $\begin{array}{l}\text { ibuprofen, } \\
\text { diclofenac-sodium, } \\
\text { ketoprofen, } \\
\text { naproxen, }\end{array}$ & $\begin{array}{l}\text { stainless } \\
\text { steel }\end{array}$ & 12 & 1.5 & natural soil & $\mathrm{n} / \mathrm{a}$ & $\begin{array}{l}0.42-0.48 \\
\text { (calcu- } \\
\text { lated us- } \\
\text { ing pro- } \\
\text { vided } \\
\text { mass, bulk } \\
\text { density } \\
\text { and pore } \\
\text { volume) }\end{array}$ & $n / a$ & $\begin{array}{l}\text { aluminum } \\
\text { plate with } \\
\text { a stain- } \\
\text { less steel } \\
\text { needle }\end{array}$ & acrylic & by gravity & $\begin{array}{l}\text { artificial } \\
(\text { ground) water } \\
\left(\mathrm{CaCl}_{2}\right) \\
\text { deionized water, } \\
\text { DOC and poly- } \\
\text { acrylamide } \\
\text { amendment }\end{array}$ & $\begin{array}{l}\text { about } 2 \\
\mathrm{mg} / \mathrm{kg} \\
\text { (sub- } \\
\text { stance / } \\
\text { soil) }\end{array}$ & $\mathrm{n} / \mathrm{a}$ & manual \\
\hline $\begin{array}{l}\text { Yao et al. } \\
(2012)\end{array}$ & leaching & sulfamethoxazole & $\begin{array}{l}\text { acrylic } \\
\text { cylinder }\end{array}$ & 16.5 & 4 & $\begin{array}{l}\text { natural soil, } \\
\text { artificial sedi- } \\
\text { ment (biochar } \\
\text { \& sandy soil) }\end{array}$ & $\begin{array}{l}\text { wet } \\
\text { (satu- } \\
\text { rated) }\end{array}$ & $\mathrm{n} / \mathrm{a}$ & $n / a$ & $\begin{array}{l}\text { stainless } \\
\text { steel } \\
\text { mesh }\end{array}$ & $n / a$ & $\mathrm{n} / \mathrm{a}$ & $\begin{array}{l}\text { artificial } \\
\text { (ground)water, } \\
\text { artificial re- } \\
\text { claimed water }\end{array}$ & $2 \mathrm{mg} / \mathrm{L}$ & $n / a$ & manual \\
\hline
\end{tabular}




\section{References}

Aga, D. S., Goldfish, R., and Kulshrestha, P.: Application of ELISA in determining the fate of tetracyclines in land-applied livestock wastes, Analyst, 128, 658-662, 10.1039/b301630g, 2003.

Alidina, M., Li, D., Ouf, M., and Drewes, J. E.: Role of primary substrate composition and concentration on attenuation of trace organic chemicals in managed aquifer recharge systems, J. Environ. Manag., 144, 58-66, 2014.

Alotaibi, M. D., Patterson, B. M., McKinley, A. J., Reeder, A. Y., Furness, A. J., and Donn, M. J.: Fate of benzotriazole and 5-methylbenzotriazole in recycled water recharged into an anaerobic aquifer: Column studies, Water Res., 70, 184-195, 10.1016/j.watres.2014.11.040, 2015.

Banzhaf, S., Nödler, K., Licha, T., Krein, A., and Scheytt, T.: Redox-sensitivity and mobility of selected pharmaceutical compounds in a low flow column experiment, Sci. Tot. Environ., 438, 113-121, 10.1016/j.scitotenv.2012.08.041 2012.

Baumgarten, B., Jahrig, J., Reemtsma, T., and Jekel, M.: Long term laboratory column experiments to simulate bank filtration: Factors controlling removal of sulfamethoxazole, Water Res., 45, 211-220, 10.1016/j.watres.2010.08.034, 2011.

Bertelkamp, C., Reungoat, J., Botton, S., Cornelissen, E., Ghadiri, E., de Jonge, M., Singhal, N., van der Hoek, J. P., and Verliefde, A. R. D.: Transformation of organic micropollutants during river bank filtration: Laboratory versus field data, Water Pract. Technol., 7, 10.2166/wpt.2012.081, 2012.

Bertelkamp, C., Reungoat, J., Cornelissen, E. R., Singhal, N., Reynisson, J., Cabo, A. J., van der Hoek, J. P., and Verliefde, A. R. D.: Sorption and biodegradation of organic micropollutants during river bank filtration: A laboratory column study, Water Res., 52, 231-241, 10.1016/j.watres.2013.10.068, 2014.

Burke, V., Treumann, S., Duennbier, U., Greskowiak, J., and Massmann, G.: Sorption behavior of 20 wastewater originated micropollutants in groundwater - Column experiments with pharmaceutical residues and industrial agents, J. Contam. Hydrol., 154, 29-41, 10.1016/j.jconhyd.2013.08.001, 2013.

Burke, V., Greskowiak, J., Asmuß, T., Bremermann, R., Taute, T., and Massmann, G.: Temperature dependent redox zonation and attenuation of wastewater-derived organic micropollutants in the hyporheic zone, Sci. Tot. Environ., 482, 53-61, 2014.

Burke, V., Greskowiak, J., Grünenbaum, N., and Massmann, G.: Redox and temperature dependent attenuation of 20 organic micropollutants-a systematic column study, Water Environ. Res., 2016.

Cabrera-Lafaurie, W. A., Román, F. R., and Hernández-Maldonado, A. J.: Single and multi-component adsorption of salicylic acid, clofibric acid, carbamazepine and caffeine from water onto transition metal modified and partially calcined inorganic-organic pillared clay fixed beds, J. Hazard. Mater., 282, 174-182, 2015.

Casas, M. E., and Bester, K.: Can those organic micro-pollutants that are recalcitrant in activated sludge treatment be removed from wastewater by biofilm reactors (slow sand filters)?, Sci. Tot. Environ., 506, 315-322, 2015.

Chen, H., Gao, B., Li, H., and Ma, L. Q.: Effects of pH and ionic strength on sulfamethoxazole and ciprofloxacin transport in saturated porous media, J. Contam. Hydrol., 126, 2936, 2011.

Chiang, W.-H., and Kinzelbach, W.: Processing Modflow, A simulation program for modelling groundwater flow and pollution. User manual, 1998.

Cordy, G. E., Duran, N. L., Bouwer, H., Rice, R. C., Furlong, E. T., Zaugg, S. D., Meyer, M. T., Barber, L. B., and Kolpin, D. W.: Do pharmaceuticals, pathogens, and other organic waste water compounds persist when waste water is used for recharge?, Ground Water Monit. Rem., 24, 58-69, 2004.

D'Alessio, M., Yoneyama, B., and Ray, C.: Fate of selected pharmaceutically active compounds during simulated riverbank filtration, Sci. Tot. Environ., 505, 615-622, 2015. De Wilde, T., Mertens, J., Simunek, J., Sniegowksi, K., Ryckeboer, J., Jaeken, P., Springael, D., and Spanoghe, P.: Characterizing pesticide sorption and degradation in microscale biopurification systems using column displacement experiments, Environ. Pollut., 157, 463-473, 10.1016/j.envpol.2008.09.008, 2009.

Doherty, J.: PEST: Model-independent parameter estimation, Watermark Computing, Corinda, Australia, 2002.

Doherty, J.: PEST: Model-independent parameter estimation, 5th edition, Watermark Computing, Corinda, Australia, 2005. 
Dusek, J., Dohnal, M., Snehota, M., Sobotkova, M., Ray, C., and Vogel, T.: Transport of bromide and pesticides through an undisturbed soil column: A modeling study with global optimization analysis, J. Contam. Hydrol., 175-176, 1-16, 10.1016/j.jconhyd.2015.02.002, 2015.

Estrella, M. R., Brusseau, M. L., Maier, R. S., Pepper, I. L., Wierenga, P. J., and Miller, R. M.: Biodegradation, sorption, and transport of 2,4-dichlorophenoxyacetic acid in saturated and unsaturated soils, Appl. Environ. Microbiol., 59, 4266-4273, 1993.

Fan, Z., Casey, F. X. M., Hakk, H., Larsen, G. L., and Khan, E.: Sorption, Fate, and Mobility of Sulfonamides in Soils, Water Air Soil Pollut., 218, 49-61, 10.1007/s11270-0100623-6, 2011.

Frisch, M., Trucks, G., Schlegel, H., Scuseria, G., Robb, M., Cheeseman, J., Montgomery Jr, J., Vreven, T., Kudin, K., and Burant, J.: Gaussian 03, revision C. 02, Gaussian, Inc., Wallingford, CT, 2004

Greenhagen, A. M., Lenczewski, M. E., and Carroll, M.: Natural attenuation of pharmaceuticals and an illicit drug in a laboratory column experiment, Chemosphere, 115, 13-19, 2014.

Gruenheid, S., Huebner, U., and Jekel, M.: Impact of temperature on biodegradation of bulk and trace organics during soil passage in an indirect reuse system, Water Sci. Technol., 57, 987-994, 10.2166/wst.2008.207, 2008.

Hebig, K. H., Groza, L. G., Sabourin, M. J., Scheytt, T. J., and Ptacek, C. J.: Transport behavior of the pharmaceutical compounds Carbamazepine, Sulfamethoxazole, Gemfibrozil, Ibuprofen, and Naproxen, and of the lifestyle drug Caffeine in saturated laboratory columns, Sci. Tot. Environ., submitted.

Ji, X., and Xu, Y.: libSRES: A C library for stochastic ranking evolution strategy for parameter estimation, Bioinformatics, 22, 124-126, 10.1093/bioinformatics/bti753, 2006. Jia, Y., Breedveld, G. D., and Aagaard, P.: Column studies on transport of deicing additive benzotriazole in a sandy aquifer and a zerovalent iron barrier, Chemosphere, 69, 1409-1418, 10.1016/j.chemosphere.2007.04.074, 2007.

Jury, W. A., and Sposito, G.: Field calibration and validation of solute transport models for the unsaturated zone, Soil Sci. Soc. Am. J., 49, 1331-1341, 1985.

Kamra, S. K., Lennartz, B., Van Genuchten, M. T., and Widmoser, P.: Evaluating non-equilibrium solute transport in small soil columns, J. Contam. Hydrol., 48, $189-212,2001$.

Kay, P., Blackwell, P. A., and Boxall, A. B. A.: Column studies to investigate the fate of veterinary antibiotics in clay soils following slurry application to agricultural land, Chemosphere, 60, 497-507, 10.1016/j.chemosphere.2005.01.028, 2005.

Ke, J., Gin, K. Y. H., Tan, L. H., and Reinhard, M.: Fate of endocrine-disrupting and pharmaceutically active substances in sand columns fed with secondary effluent, Journal of Environmental Engineering (United States), 138, 1067-1076, 10.1061/(ASCE)EE.1943-7870.0000564, 2012.

Lopez-Blanco, M. C., Cancho-Grande, B., Simal-Gandara, J., Lopez-Periago, E., and Arias-Estevez, M.: Transport of commercial endosulfan through a column of aggregated vineyard soil by a water flux simulating field conditions, J. Agric. Food Chem., 53, 6738-6743, 10.1021/jf050545i, 2005.

Lorphensri, O., Sabatini, D. A., Kibbey, T. C. G., Osathaphan, K., and Saiwan, C.: Sorption and transport of acetaminophen, 17 alpha-ethynyl estradiol, nalidixic acid with low organic content aquifer sand, Water Res., 41, 2180-2188, 10.1016/j.watres.2007.01.057, 2007.

Maeng, S. K., Sharma, S. K., Abel, C. D. T., Magic-Knezev, A., and Amy, G. L.: Role of biodegradation in the removal of pharmaceutically active compounds with different bulk organic matter characteristics through managed aquifer recharge: Batch and column studies, Water Res., 45, 4722-4736, 10.1016/j.watres.2011.05.043, 2011.

Massmann, G., Dünnbier, U., Heberer, T., and Taute, T.: Behaviour and redox sensitivity of pharmaceutical residues during bank filtration - Investigation of residues of phenazone-type analgesics, Chemosphere, 71, 1476-1485, 10.1016/j.chemosphere.2007.12.017, 2008.

Mersmann, P., Scheytt, T., and Heberer, T.: Column experiments on the transport behavior of pharmaceutically active compounds in the saturated zone, Acta Hydroch. Hydrob., 30, 275-284, 2002.

Microcal Software Inc.: Origin Users's Manual, Microcal Software, Inc., Northampton, USA, 1995.

Murillo-Torres, R., Durán-Álvarez, J. C., Prado, B., and Jiménez-Cisneros, B. E.: Sorption and mobility of two micropollutants in three agricultural soils: A comparative analysis of their behavior in batch and column experiments, Geoderma, 189-190, 462-468, 10.1016/j.geoderma.2012.05.019, 2012. 


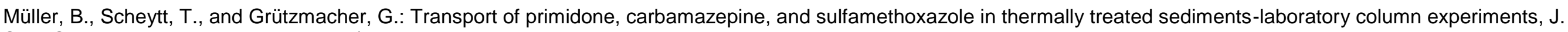
Soils Sediments, 13, 953-965, 10.1007/s11368-013-0671-9, 2013.

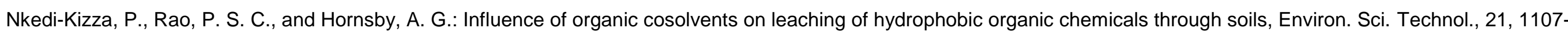
1111, 1987.

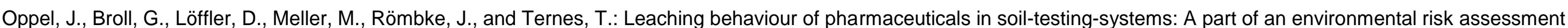
for groundwater protection, Sci. Tot. Environ., 328, 265-273, 10.1016/j.scitotenv.2004.02.004, 2004.

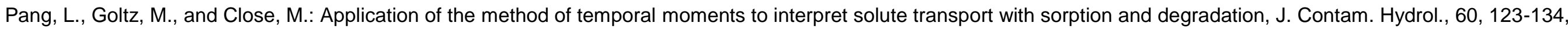
10.1016/S0169-7722(02)00061-X, 2003.

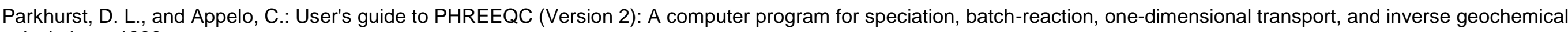
calculations, 1999.

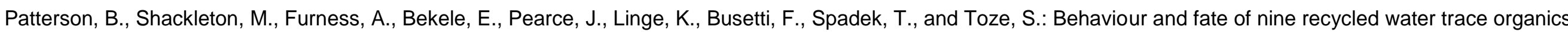
during managed aquifer recharge in an aerobic aquifer, J. Contam. Hydrol., 122, 53-62, 2011.

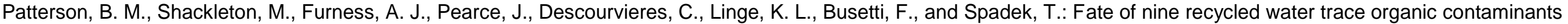
and metal(loid)s during managed aquifer recharge into a anaerobic aquifer: Column studies, Water Res., 44, 1471-1481, 10.1016/j.watres.2009.10.044, 2010.

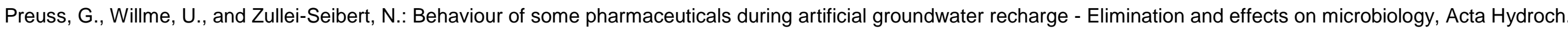
Hydrob., 29, 269-277, 2001.

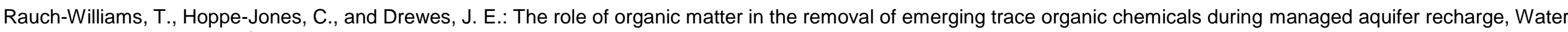
Res., 44, 449-460, 10.1016/j.watres.2009.08.027, 2010.

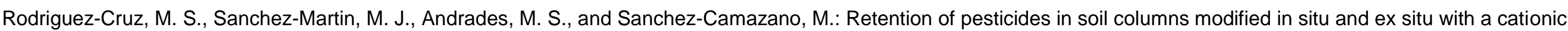
surfactant, Sci. Tot. Environ., 378, 104-108, 10.1016/j.scitotenv.2007.01.021, 2007.

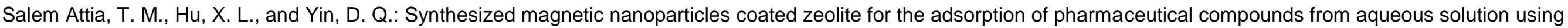
batch and column studies, Chemosphere, 93, 2076-2085, 10.1016/j.chemosphere.2013.07.046, 2013.

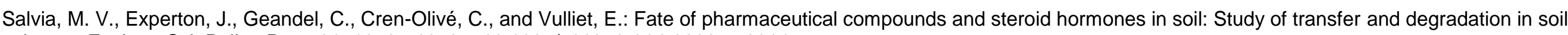
columns, Environ. Sci. Pollut. Res., 21, 10525-10535, 10.1007/s11356-014-3038-x, 2014.

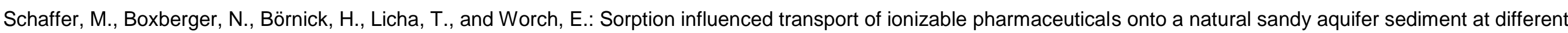
$\mathrm{pH}$, Chemosphere, 87, 513-520, 10.1016/j.chemosphere.2011.12.053, 2012a.

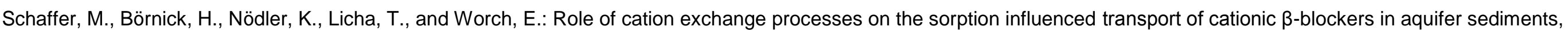
Water Res., 46, 5472-5482, $2012 \mathrm{~b}$.

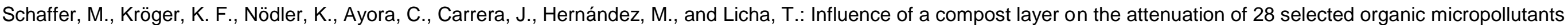
under realistic soil aquifer treatment conditions: Insights from a large scale column experiment, Water Res., 74, 110-212, 10.1016/j.watres.2015.02.010, 2015.

Scheytt, T., Grams, S., and Fell, H.: Vorkommen und Verhalten eines Arzneimittels (Clofibrinsäure) im Grundwasser, Grundwasser, 2/98, 67-77, 1998.

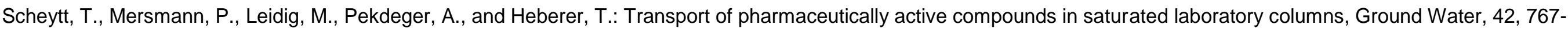
773, 2004.

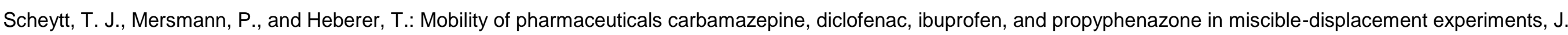
Contam. Hydrol., 83, 53-69, 2006.

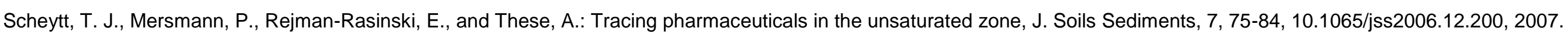


Siemens, J., Huschek, G., Walshe, G., Siebe, C., Kasteel, R., Wulf, S., Clemens, J., and Kaupenjohann, M.: Transport of Pharmaceuticals in Columns of a Wastewater-Irrigated Mexican Clay Soil, J. Environ. Qual., 39, 1201-1210, 10.2134/jeq2009.0105, 2010.

Simon, R., Colón, D., Tebes-Stevens, C. L., and Weber, E. J.: Effect of redox zonation on the reductive transformation of p-cyanonitrobenzene in a laboratory sediment column, Environ. Sci. Technol., 34, 3617-3622, 10.1021/es000960l, 2000.

Simunek, J., Huang, K., and Van Genuchten, M. T.: The HYDRUS code for simulating the one-dimensional movement of water, heat, and multiple solutes in variably-saturated media, US Salin. Lab. Res. Rep, 144, 1998.

Simunek, J., Van Genuchten, M. T., and Sejna, M.: The HYDRUS-1D software package for simulating the movement of water, heat, and multiple solutes in variably saturated media, version 3.0, HYDRUS software series 1, Department of Environmental Sciences, University of California Riverside, Riverside Edition, 2005.

Šimůnek, J., and Van Genuchten, M. T.: Modeling nonequilibrium flow and transport processes using HYDRUS, Vadose Zone J., 7, 782-797, 10.2136/vzj2007.0074, 2008. Srivastava, P., Sanders, S. M., Dane, J. H., Feng, Y., Basile, J., and Barnett, M. O.: Fate and Transport of Sulfadimethoxine and Ormetoprim in Two Southeastern United States Soils, Vadose Zone J., 8, 32-41, 10.2136/vzj2007.0186, 2009.

Strauss, C., Harter, T., and Radke, M.: Effects of pH and Manure on Transport of Sulfonamide Antibiotics in Soil, J. Environ. Qual., 40, 1652-1660, 10.2134/jeq2010.0535, 2011. Teijón, G., Candela, L., Šimůnek, J., Tamoh, K., and Valdes-Abellán, J.: Fate and Transport of Naproxen in a Sandy Aquifer Material: Saturated Column Studies and Model Evaluation, Soil Sediment Contam., 23, 736-750, 10.1080/15320383.2014.869194, 2014.

Toride, N., Leii, F. J., and van Genuchten, M. T.: The CXTFIT code for estimating transport parameters from laboratory or field tracer experiments, Version 2.0, U.S. Salinity Laboratory, USDA, ARS, Riverside, California., 1995.

Toride, N., Leij, F. J., and van Genuchten, M. T.: The CXTFIT code for estimating transport parameters from laboratory or field tracer experiments, Version 2.1, U.S. Salinity Laboratory, USDA, ARS, Riverside, California., 1999.

University of Florida: UFBTC, version 2-One dimensional subsurface transport model, finite difference technique, 1989.

Unold, M., Kasteel, R., Groeneweg, J., and Vereecken, H.: Transport and transformation of sulfadiazine in soil columns packed with a silty loam and a loamy sand, J. Contam. Hydrol., 103, 38-47, 10.1016/j.jconhyd.2008.09.002, 2009.

Van Genuchten, M. T.: Convective-dispersive transport of solutes involved in sequential first-order decay reactions, Computers and Geosciences, 11, 129-147, 10.1016/00983004(85)90003-2, 1985.

Van Genuchten, M. T., and Wagenet, R.: Two-site/two-region models for pesticide transport and degradation: Theoretical development and analytical solutions, Soil Sci. Soc. Am. J., 53, 1303-1310, 1989

Vogel, T., Lichner, L., Dusek, J., and Cipakova, A.: Dual-continuum analysis of a cadmium tracer field experiment, J. Contam. Hydrol., 92, 50-65, 10.1016/j.jconhyd.2007.01.001, 2007 .

Wu, C., Spongberg, A. L., Witter, J. D., Fang, M., Czajkowski, K. P., and Ames, A.: Dissipation and leaching potential of selected pharmaceutically active compounds in soils amended with biosolids, Arch. Environ. Contam. Toxicol., 59, 343-351, 2010.

Xu, J., Wu, L. S., Chen, W. P., and Chang, A. C.: Leaching potential of nonsteroidal anti-inflammatory drugs in soils, Environ. Toxicol. Chem., 29, 800-807, 10.1002/etc.107, 2010

Yao, Y., Gao, B., Chen, H., Jiang, L., Inyang, M., Zimmerman, A. R., Cao, X., Yang, L., Xue, Y., and Li, H.: Adsorption of sulfamethoxazole on biochar and its impact on reclaimed water irrigation, J. Hazard. Mater., 209, 408-413, 10.1016/j.jhazmat.2012.01.046, 2012. 